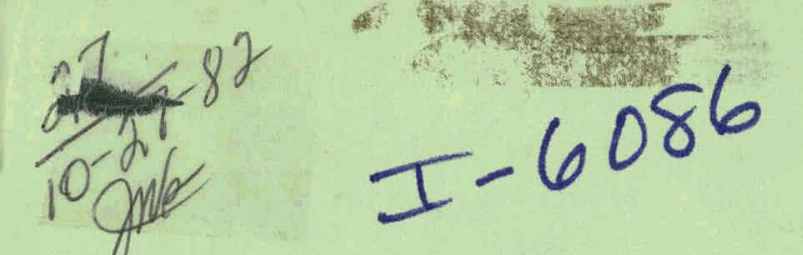

UCID-19577

\title{
HOT ELECTRONS IN THE ANCHOR REGION \\ OF THE AXTCELL DESIGN OF THE \\ MIRROR FUSION TEST FACILITY (MFTF)
}

J. W. Shearer

September 14, 1982

This is an informal report intended primarily for internal or Ilmited external distributiun. The opinions and conclusions stated are those of the author and may or may not be those of the Laboratory.

Work performed under the auspices of the U.S. Department of Energy by the Lawrence Livermore Laboratory under Contract W-7405-Eng-48. 


\section{DISCLAIMER}

This report was prepared as an account of work sponsored by an agency of the United States Government. Neither the United States Government nor any agency Thereof, nor any of their employees, makes any warranty, express or implied, or assumes any legal liability or responsibility for the accuracy, completeness, or usefulness of any information, apparatus, product, or process disclosed, or represents that its use would not infringe privately owned rights. Reference herein to any specific commercial product, process, or service by trade name, trademark, manufacturer, or otherwise does not necessarily constitute or imply its endorsement, recommendation, or favoring by the United States Government or any agency thereof. The views and opinions of authors expressed herein do not necessarily state or reflect those of the United States Government or any agency thereof. 


\section{DISCLAIMER}

Portions of this document may be illegible in electronic image products. Images are produced from the best available original document. 
HOT ELECTRONS IN THE ANCHOR REGION OF THE AXICELL DESIGN OF THE MIRROR FUSION TEST. FACILITY (MFTF)

J. W. Shearer

Lawrence Livermore National Laboratory, University of California

Livermore, CA 94550

\section{ABSTRACT}

The axicell design of the Mirror Fusion Test Facility (MFTF) requires electron cyclotron resonance heating (ECRH) up to average electron energies of as high as $450 \mathrm{keV}$. These temperatures, plus the magnetic field and plasma beta profiles, lead to the requirement for three frequencies--28, 35, and 56 (or 60) GHz. Power balance studies include the effects of scattering, drag, synchrotron radiation, and cold electron production, and they predict that about $600 \mathrm{~kW}$ of ECRH power is needed at each end of MFTF.

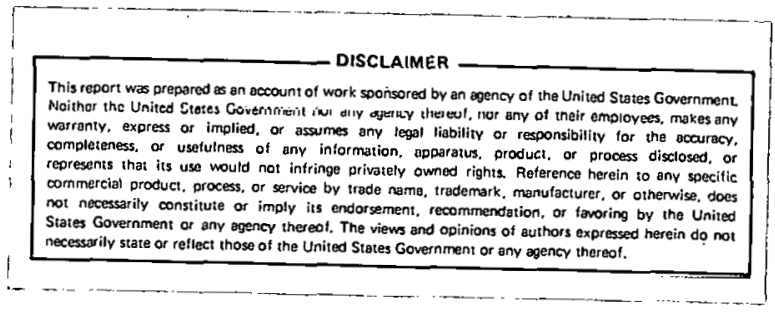




\section{THIS PAGE \\ WAS INTENTIONALLY \\ LEFT BLANK}


1. Introduction

2. Energy $(\varepsilon)$ and Magnetic Moment $(\mu)$ Coordinates 1

3. Frequencies of ECRH 9

4. Hot Electron Scattering Losses 13

5. Fokker-Planck Scattering Results 18

6. Electron-Electron Drag . 23

7. Radiation Losses , 25

8. Summary of Hot Electron Losses 26

9. Hot Electron Power Loss for a Simple Model 27

10. Power Estimates for the Warm Electrons 31

11. ECRH Requirements for MFTF Axicell Design 35

12. Acknowledgments 35

References 38

Appendix A. Effective Area and Length Integrals 40 


\section{ILLUSTRATIONS}

Fig. 1. Computer plot of one end of an MFTF axicell design, showing (from left to right) the two yin-yang coils, the two transition coils, the two axicell coils, and six of the twelve solenoid coils.

Fig. 2. Approximate field and potential profiles expected for the transition and anchor sections of one end of the MFTF axicell design. The six labeled points provide the input parameters for the next figure.

Fig. 3. Energy and magnetic moment ( $\varepsilon$ ys $\mu$ ) plot of electrons for the points shown in Fig. 2. The hot electron region is labeled.

F1g. 4. Same as Fig. 3 with expanded scales to emphasize the regions for the warm electrons and the passing electrons.

Fig. 5. Magnetic field and electron density profiles in the anchor region. $B_{v} \equiv$ vacuum field, $B_{p} \equiv$ beta-depressed field, $n_{p} \equiv$ passing electrons, $n_{h} \equiv$ hot electrons, $n_{w} \equiv$ warm electrons. (a) and (b) are ECRH heating locations.

Fig. 6. Variation of resonant field $B_{R E S}$ and plasma field $B_{p}$ with electron energy $E_{e}$ for the fundamental $(n=1)$ and the harmonic $(n=2)$ of two gyrotron frequencies at point (a) of $\mathrm{Fig} .5$.

Fig. 7. Same as Fig. 6 for point (b) of Fig. 5, and for the harmonics of the highest gyrotron frequency.

Fig. 8. Relativistic corrections to the power equations for scattering losses and drag losses.

Fig. 9. Variation of current and energy constants for nine Fokker Planck code runs as calculated from Eqs. (5.2) and (5.4).

Fig. 10. Comparison of the current constant $K_{I}$ with the stream temperature $T_{e s}$, using $\mathrm{Eq} .(5.5)$ with $\mathrm{n}_{\mathrm{s}}=2 \times 10^{11}$.

Fig. 11. Time dependence of electron density, average energy, $r f$ power, losses, current constant $K_{I}$, energy constant $K_{E}$, and the product $K_{I} K_{E}$ plotted for one MFTF-related problem on the Fokker-Planck code.

Fig. 12. Approximate linear model of electron density distributions in a quadratic magnetic well--for use in estimating electron power losses, based roughly on the profiles in Fig. 5 .

Fig. 13. Hot electron loss powers versus electron energy in the MFTF anchor model calculation at constant beta. 


\section{TABLES}

Table 1. Common parameters of the comparison.

Table 2. Characteristic parameters of the anchor region of two experiments.

Table 3. Power losses of hot electrons in each anchor.

Table 4. Parameters for MFTF anchor warm electron heating.

Table 5. ECRH power distribution for each anchor region of the MFTF axicell. 
THIS PAGE

\section{WAS INTENTIONALLY \\ LEFT BLANK}




\section{INTRODUCTION}

The end-plug design in the Mirror Fusion Test Facility (MFTF) was recently changed to an axicell configuration, ${ }^{1}$ and a physics model for the plasma parameters of this new configuration has been developed. ${ }^{2}$ Figure 1 shows the new magnet configuration, and Fig. 2 shows typical profiles of the axial dependence of the magnetic field and electrostatic potential.

In the MFTF axicell design both the electron thermal barrier and the ion plug potential are located in the minimum-B quadrupole field of the "anchor" region. Therefore, all of the electron cyclotron resonance heating (ECRH) must be located there. The ECRH must establish and maintain an average hot electron energy of $\simeq 450 \mathrm{keV}$ in the thermal barrier and a warm electron temperature of $\simeq 70 \mathrm{keV}$ in the plug. ${ }^{2}$

This report elaborates on the expected physical properties of the electrons in each anchor region, with emphasis on the identification of the necessary parameters for the ECRH hardware.

\section{ENERGY $(\varepsilon)$ AND MAGNETIC MOMENT $(\mu)$ COORDINATES}

$\varepsilon-\mu$ coordinates are in common use for MFTF calculations; they are especially useful for relativistic electrons, including the relativistic Fokker-Planck code presently under development. ${ }^{3}$ Velocity coordinates would be unsuitable for relativistic particles, since the phase space would crowd up against the limit $v=c$. Momentum coordinates would be better, but they are not as easily visualized as $\varepsilon-\mu$ coordinates, because they are not linear in either energy or velocity.

Consider the mumentum $\mu$ :

$$
p=m v=r m_{0} v \text {. }
$$

Here $m_{0}$ is the rest mass, and $\gamma$ is the relativistic energy parameter

$$
\gamma=1+(\varepsilon-\Phi) / m_{0} c^{2}
$$

where $\varepsilon-\Phi$ is the kinetic energy, and $\Phi$ is the electrostatic potential

energy. The relativistic relation between $\gamma$ and $p$ is:

$$
r^{2}=1+\left(\frac{p}{m_{0} c}\right)^{2} \text {. }
$$




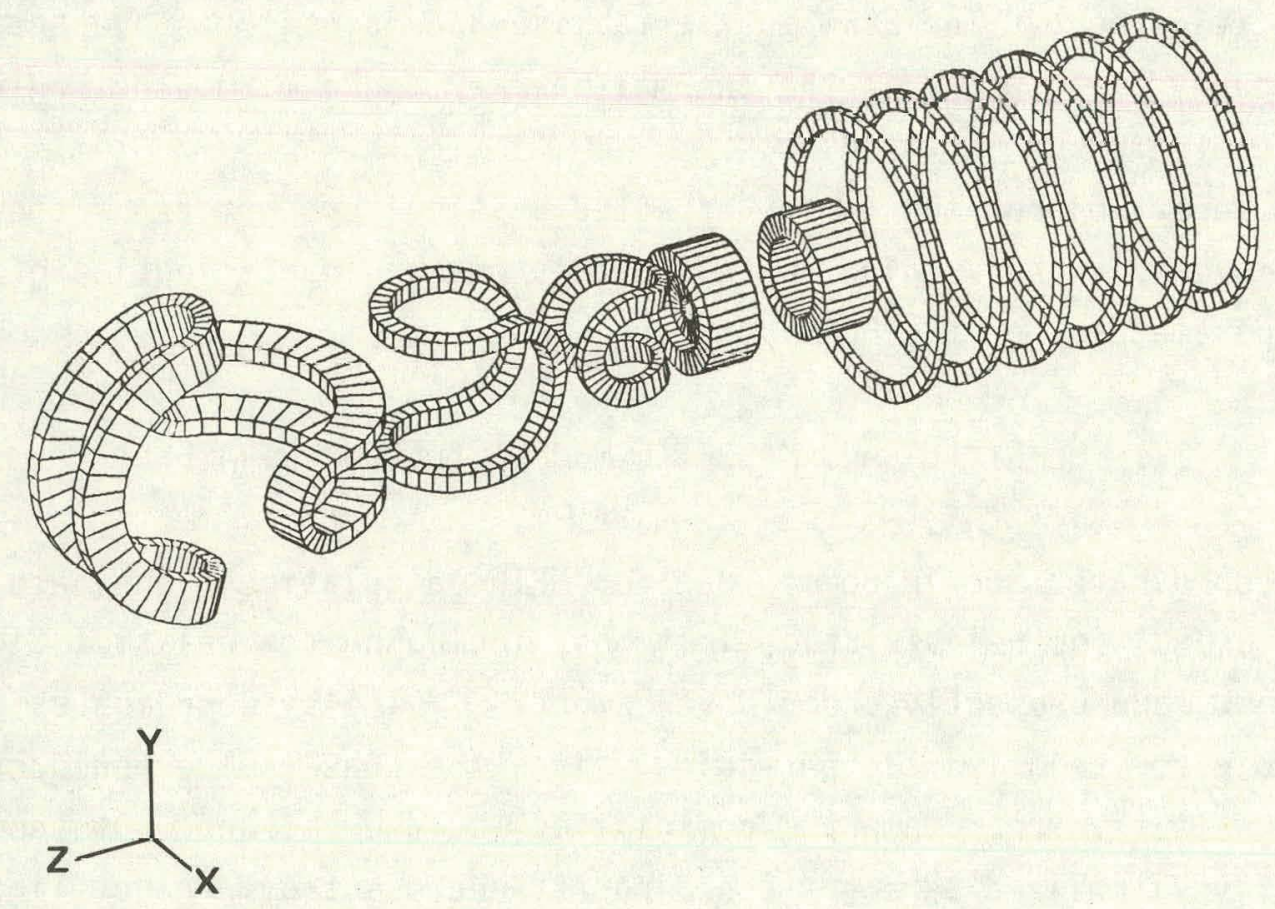

Figure 1. Computer plot of one end of an MFTF axicell design, showing (from left to right) the two yin-yang coils, the two transition coils, the two axicell coils, and six of the twelve solenoid coils. 

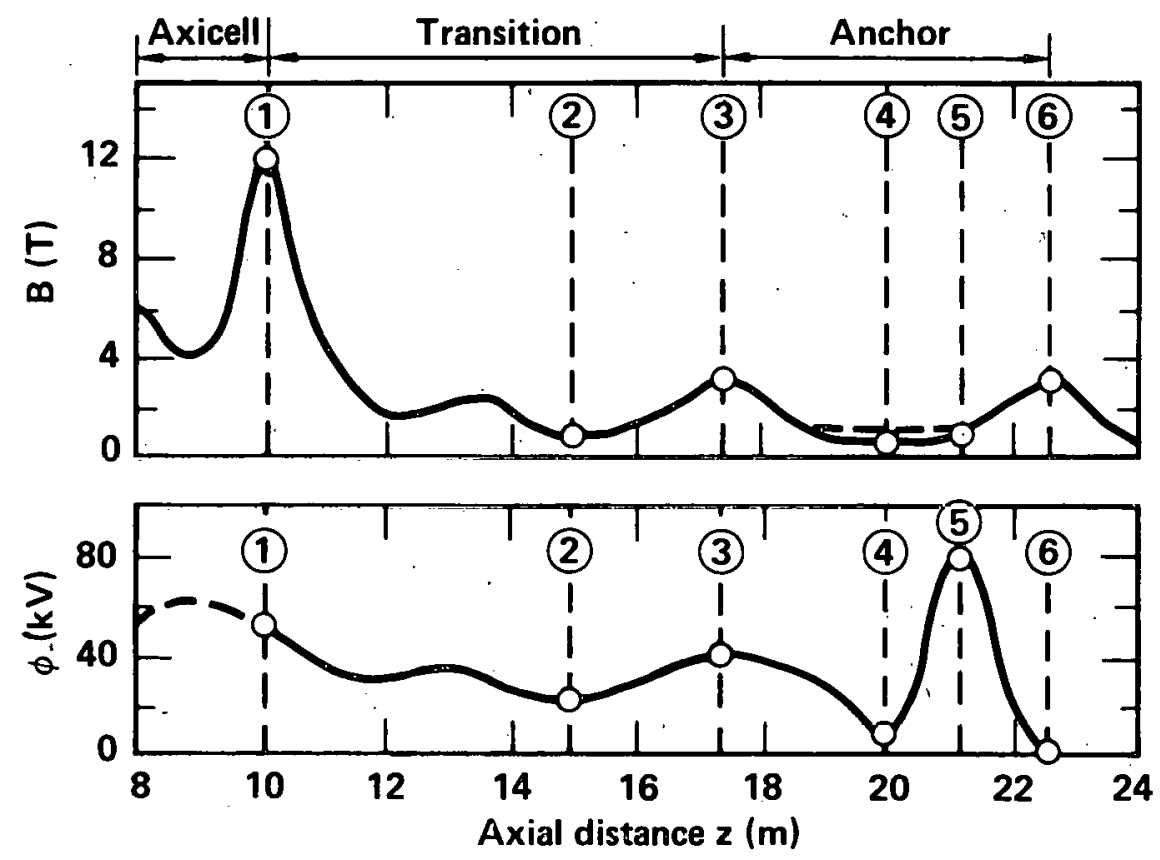

Figure 2. Approximate field and potential profiles expected for the transition and anchor sections of one end of the MFTF axicell design. The six labeled points provide the input parameters for the next figure. 
We divide the momentum into two components

$$
p^{2}=p_{\|}^{2}+p_{1}^{2}
$$

and then the magnetic moment $\mu$ is:

$$
\mu=\frac{1}{2} \frac{p_{\perp}^{2}}{m_{0}^{B}} .
$$

Next substitute Eqs. (2.4) and (2.5) into Eq. (2.3)

$$
\gamma^{2}=1+\left(\frac{p_{\| !}}{m_{n^{r}}}\right)^{2}+\mu^{\prime} B
$$

where we have renormalized the magnetic moment in terms of the relativistic parameler $\mu^{\prime}$

$$
\mu^{\prime}=2 \mu / m_{0} c^{2}
$$

Now we are ready to derive the equation for the curve in $\varepsilon-\mu$ space which describes all those particles whose turning point occurs at the specified field $B_{S}$ and potential $\Phi_{S}$. The turning point condition is $p_{\|}=0$. Then from Eq. (2.1), and Eq. (2.6)

$$
\varepsilon=m_{0} c^{2}\left[\left(1+\mu^{\prime} B_{s}\right)^{1 / 2}-1\right]-e_{s}
$$

where the potential energy $\Phi_{S}$

$$
\Phi_{S}=q \phi_{S}=-e \phi_{S}
$$

because the electron charge is negative. If we use units of keV and tesla, then Eq. (2.8) is:

$$
\varepsilon=511\left[\left(1+\mu B_{s} / 255.5\right)^{1 / 2}-1\right]-\phi_{s} \cdot
$$

Note that in the nonrelativistic limit $\gamma \rightarrow 1$, and Eq. (2.6) then shows that (for $p_{\|}=0$ ) $\mu^{\prime} B_{S} \ll 1$, in which case Eq. (2.8) reduces to the familiar linear form:

$$
\varepsilon(N R)=\mu B_{S}-\phi_{S}
$$


Equation (2.10) determines the curves plotted in Fig. 3 for the six locations shown in Fig. 2. The obvious curvature of the lines demonstrates the need for the relativistic formulation. The loss cone for the hot electrons is the outermost yin-yang mirror coil (1ine 6); the boundary of inaccessibility is determined by the thermal barrier point. A few hot electrons surmount the inner yin-yang coil (line 3) and traverse the transition section, but most of them are magnetically trapped inside the anchor (lines 3 and 6 ) and their peak density is located closer to line 4 because $E_{\perp}>E_{11}$.

The warm electrons and passing population regions are indicated in Fig. 4, which shows the low energy portion of the $\varepsilon-\mu$ plot on an expanded scale. The warm electron upper boundary is line 4 , due to electrostatic trapping by the thermal barriers. Note the two additional electron regions, labeled a and a'. Region a contains those passing electrons which are reflected by the outer yin-yang mirror (1ine 6); they are not reflected by the thermal barrier. (line 4), and so they sometimes overlap the real warm electron space $(z>20)$. Region a' contains trapped electrons; they are reflected by the thermal barrier (1ine 4), and thus they do not overlap the real warm electron space, even though they do overlap the warm electrons in $\varepsilon-\mu$ space.

In both Fig. 3 and Fig. 4 the boundary between the hot electrons and the warm electrons is the thermal barrier (line 4). However, the bulk of the hot electrons are expected to have a much higher magnetic moment $\mu$ than the warm electrons. There is also a boundary between the hot electrons and the passing electrons in region a, but there is no direct connection with the trapped electrons in region a', even though there is a spatial overlap for $z<20$.

Figure 5 models the distribution of the three electron populations in the anchor cell. The warm electron population is created by capture of the passing electrons, and by cold electron production. from ionization of the neutral beams. They are heated by ECRH at or near point (a) of Fig. 5. Some of these warm electrons are driven into the hot population by this ECRH. The hot electrons are mainly heated by ECRH at or near point (b) of Fig. 5 . In principle, point (b) should be located at the midplane (where $z=20$ ), but in practice it is located near $z=19.4$, as shown. This is still close enough to the center for effective heating, and it permits access to the midplane for diagnostics measurements. 


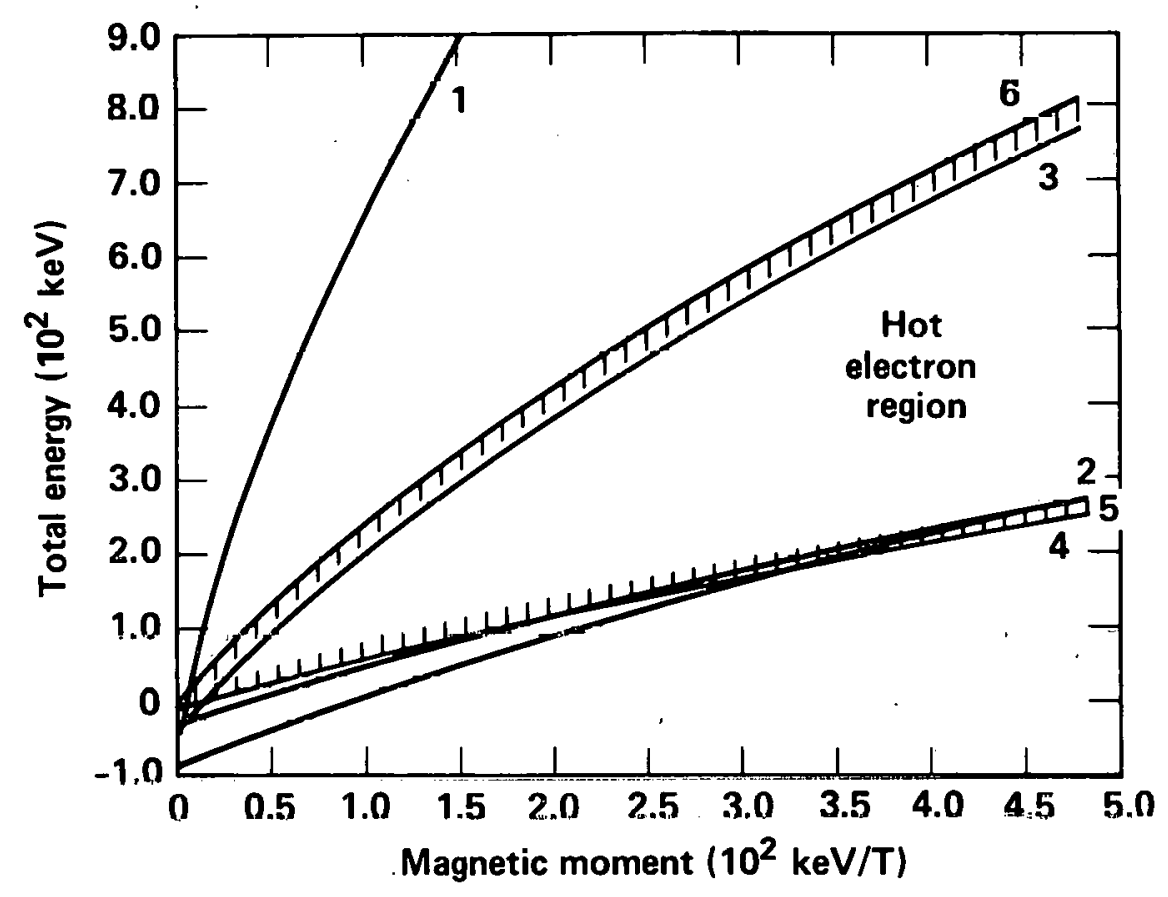

Figure 3. Energy and magnetic moment ( $\varepsilon$ vs $\mu$ ) plot of electrons for the points shown in Fig. 2. The hot electron region is labeled. 


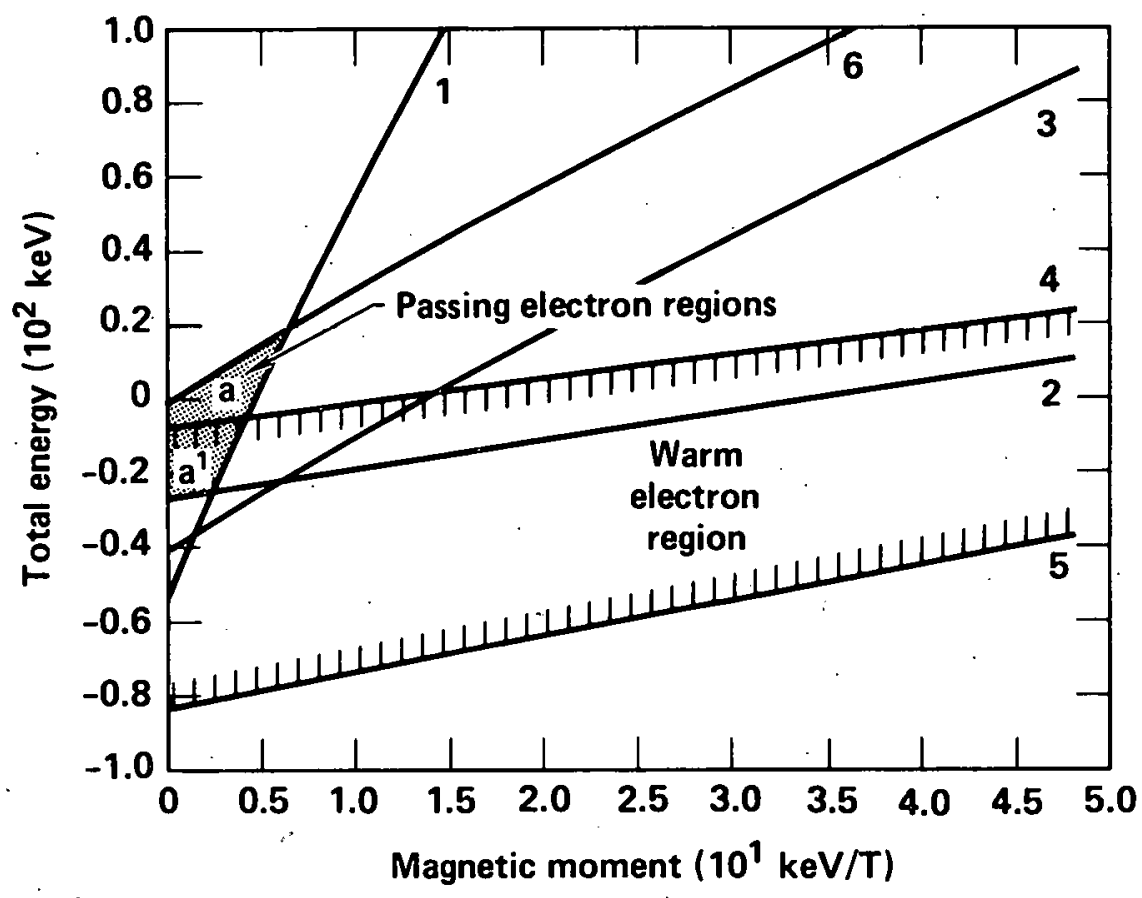

Figure 4. Same as Fig. 3 with expanded scales to emphasize the regions for the warm electrons and the passing electrons. 


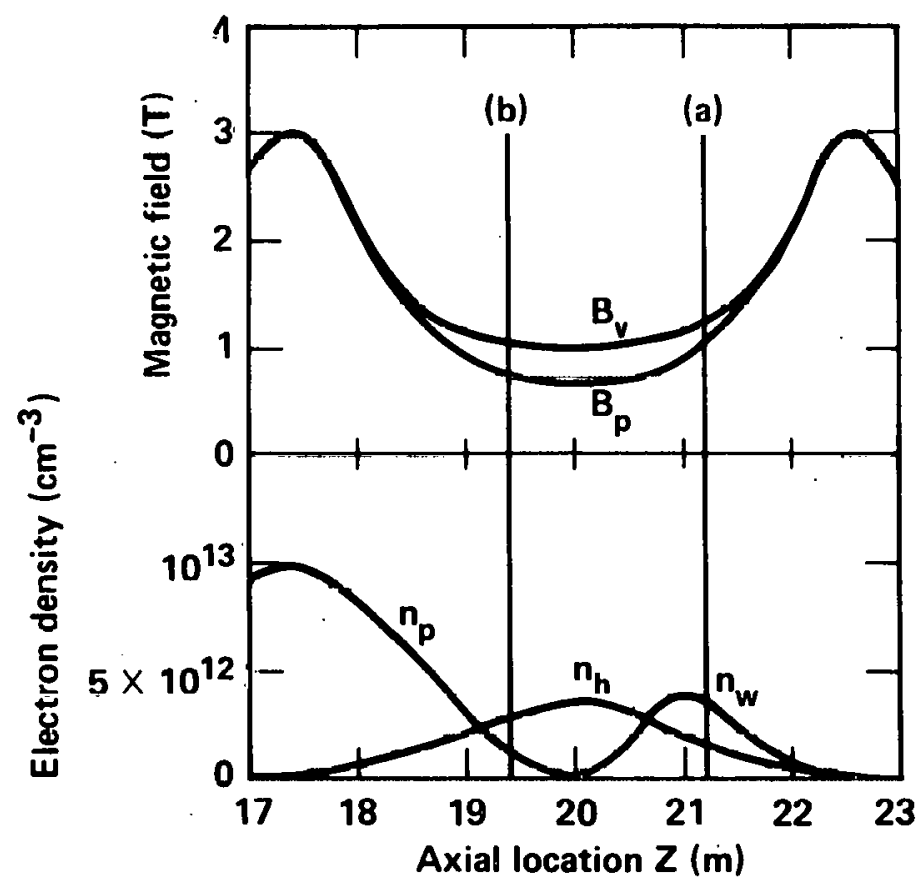

Figure 5. Magnetic field and electron density profiles in the anchor region. $B_{v} \equiv$ vacuum field, $B_{p} \equiv$ beta-depressed field, $n_{p} \equiv$ passing electrons, $n_{h} \equiv$ hot electrons, $n_{w} \equiv$ warm electrons. (a) and (b) are ECRH heating locations. 


\section{FREQUENCIES OF ECRH}

The choice of gyrotron frequencies for electron cyclotron resonance heating (ECRH) in the anchor region depends on several variables, as well as the interplay between them. They are the axial field profile ( $B$ vs $Z$ ), the temperatures $T_{h}$ and $T_{w}$ of the heated electrons, the plasma beta (which depresses the field), and the resonance field $B_{R}$ (which varies with the parallel velocity and the relativistic mass shift of the electron). A practical limitation is that only certain frequencies are available at the high power levels required for plasma heating.

Consider the model profiles of Fig. 5. The magnetic field will change during buildup of the plasma from the vacuum field $B_{v}$ to the beta-depressed field $B_{p}$

$$
B_{p}=B_{v}(1-B)^{1 / 2} \text {. }
$$

This field shift must be taken into account. The cold plasma resonance magnetic field $B_{C P R}$ is

$$
{ }^{B}{ }_{C P R}=\frac{1}{n_{v}} \frac{v(G H z)}{28}
$$

where $B$ is in tesia and $n_{v}$ is the harmonic number. As the electron temperature rises, the resonant field $B_{\text {RES }}$ changes.

$$
\frac{\mathrm{B}_{\mathrm{RES}}}{\mathrm{B}_{\mathrm{CPR}}} \simeq \gamma\left[1 \pm \cos \theta \cos \phi\left(1-\frac{1}{\gamma^{2}}\right)^{1 / 2}\right]
$$

where the relativistic gamma function is given by Eq. (2.2). The second term of Eq. (3.3) is the Doppler shift. ${ }^{5}$ Theta $(\theta)$ is the arlyle between the electron velocity vector $\underline{v}$ and the $\underline{B}$ field; phi $(\phi)$ is the angle between the wave vector $\underline{k}$ and the $\underline{B}$ field. The plasma index of refraction is assumed to be approximately unity. Equation (3.3) is another field shift that must be taken into account.

Figure 6 demonstrates the consequences of these effects for the warm electron heating at point $(a) . B_{p}$ is an approximate estimate; $B_{R E S}$ indicates the relativistic mass shift. The Doppler spread of $B_{R E S}$ is omitted for clarity; it is small at low temperatures, and leads to overlapping of the $28 \mathrm{GHz}$ and $35 \mathrm{GHz}$ resonance at high temperatures. At the start of 


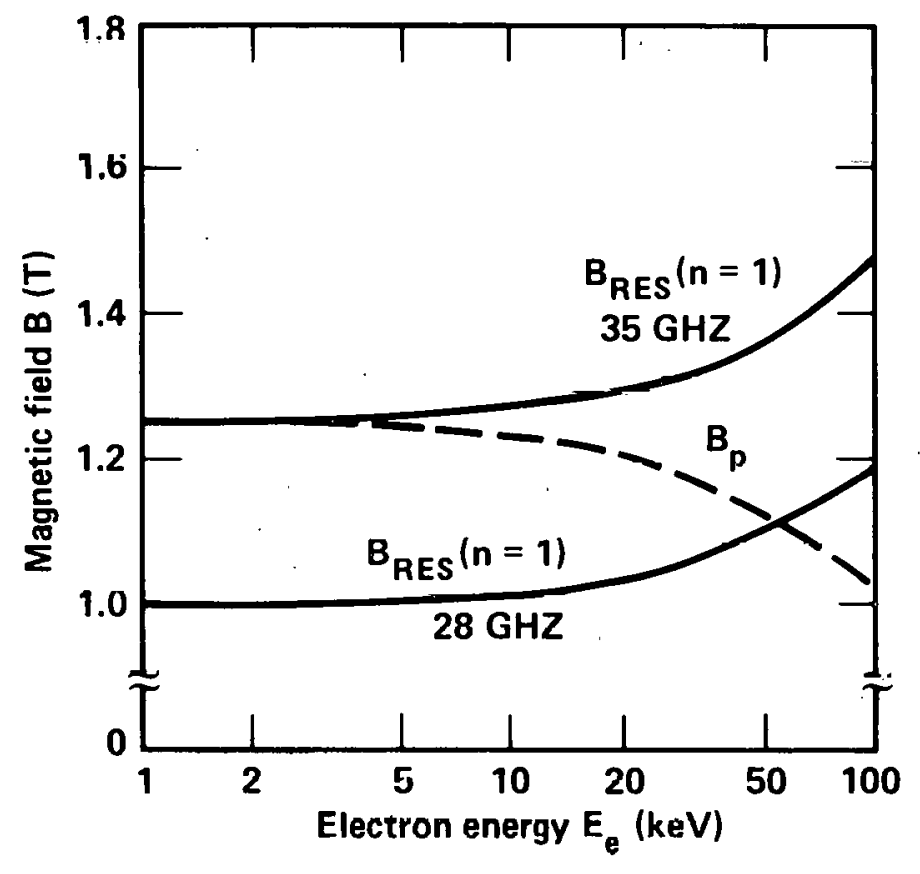

Figure 6. Variation of resonant field $B_{R E S}$ and plasma field $B_{p}$ with electron energy $E_{e}$ for the fundamental $(n=1)$ and the harmonic $(n=2)$ of two gyrotron frequencies at point (a) of Fig. 5. 
buildup, when the plasma beta and the electron temperature are both low, Fig. 6 shows that only the $35 \mathrm{GHz}$ microwaves are in resonance with the electrons. Then as the buildup proceeds, the electron temperature rises and the magnetic field decreases, until at equilibrium the $28 \mathrm{GHz}$ microwaves are in resonance with the bulk of the electrons. Details of the buildup and the transition from one frequency to another await further study. In the present state of knowledge, prudence dictates that both frequencies be specified to insure heating of all possible plasmas at this location.

Figure 7 (similar to Fig. 6) shows the same effects ( $p l$ asma beta and relativistic mass shifts) at the hot electron heating point (b). Figure 7 demonstrates that the choice of high frequency $(56 \mathrm{GHz})$ provides heating at several harmonics for the hot electrons, whose energy at equilibrium is presently expected to be in the range of 400 to $500 \mathrm{keV}$. Heating at such higher harmonics is sometimes called "off-resonant" heating, and is almost invariably used to create electron plasmas in this temperature range.6,7

The power source for ECRH is the electron cyclotron maser, usually called the "gyrotron." $28 \mathrm{GHz}$ gyrotrons are already available; $35 \mathrm{GHz}$ and $60 \mathrm{GHz}$ gyrotrons are in various stages of development, but should be ready by the time they will be needed in MFTF. 1

Both point (a) and point (b) have a gap between resonance frequency bands in the 100 to $200 \mathrm{keV}$ electron energy region. At point (a), this comes between the fundamental $\left(n_{v}=1\right) 28 \mathrm{GHz}$ resonance band and the $n_{v}=2$ harmonic of the $35 \mathrm{GHz}$ radiation. A similar resonance gap was proposed by Stallard to limit the hot electron temperature of the TMX-Upgrade experiment. ${ }^{4}$ Here, it can provide a desirable limit to the heating of the warm electrons. On the other hand, we also need to supply electrons to the hot population across this same gap. To find the right balance between these conflicting needs, one can vary the angle of incidence $\phi$, so as to change the overlapping Doppler widths of the resonances.

The corresponding gap at point (b) should not matter, provided the electron feed at point (a) is sufficient to maintain the hot electron population. Once the electron temperature exceeds $200 \mathrm{keV}$, the $n_{v} \geq 3$ harmonics of $56 \mathrm{GHz}$ at point (b) should provide sufficient heating. 


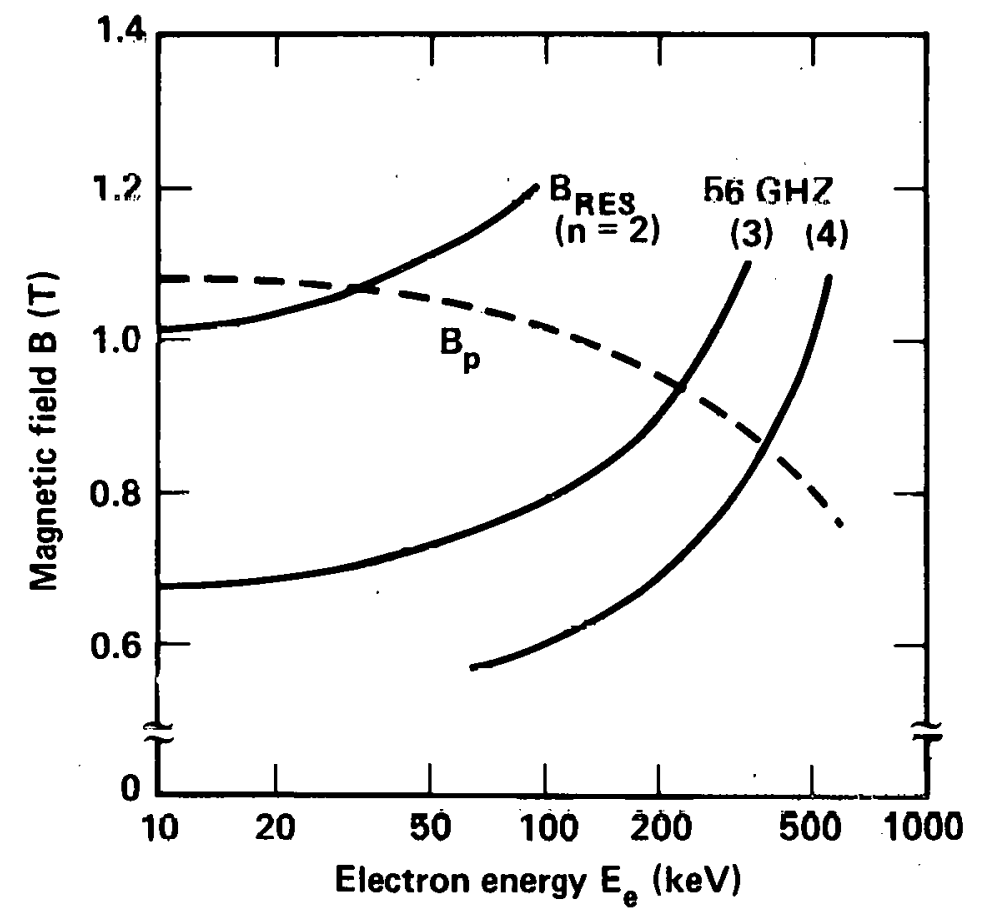

$\epsilon$

Figure 7. Same as Fig. 6 for point (b) of Fig. 5, and for the harmonics of the highest gyrotron frequency. 


\section{HOT ELECTRON SCATTERING LOSSES}

The maintenance of a hot electron cloud in the MFTF anchor cell imposes the power requirement that the ECRH absorption of microwave energy be in balance with the energy losses. These energy losses come from self-scattering, scattering from ions, scattering from cold electron populations, collisional drag by cold electron populations, and synchrotron radiation. We shall first consider the scattering losses.

Let $p_{s}^{h x}$ be the power loss of hot electrons of density $n_{h}$ and average energy $E_{h}$ by means of scattering from particles of density $n_{x}$. In cgs units, the equation for $P_{s}^{h x}$ is:

$$
P_{S}^{h x}=\left[4 \pi e^{4} \ln \Lambda(\phi-G)\right] \frac{K_{I}}{\log _{10} R_{E}} \frac{K_{E} k E_{h}}{p_{h}^{2} v_{h}} n_{h} n_{x} A_{R} L_{h x} \cdot
$$

where $k$ is Boltzmann's constant. In this equation, in $\Lambda$ and $(\phi-G)$ are well-known functions from plasma scattering theory. ${ }^{9}$ The function $\log _{10} R_{e}$ is a fit to collisional Fokker-Planck calculations in mirror geometry, ${ }^{10}$ where $R_{E}$ is the effective mirror ratio, given by

$$
R_{E} \equiv R_{M}\left(1-q \phi / k E_{h}\right)^{-1}
$$

where $R_{M}$ is the usual mirror ratio, and $\phi$ is the potential at the mirror, when $\phi=0$ at the midplane. For electrons, $q=-e$.

We define the factor $K_{I}$ as a measure of changes in the scattered electron current due to the influence of the radio frequency field. The factor $K_{E}$ represents the ratio of the average energy per scattered particle to the average energy of the trapped particle. Because the collisional scattering rate is faster for lower energy particles, $K_{E}$ is of ten put equal to one half. We might have combined $K_{I}$ and $K_{E}$ into one constant $K$, but this would have obscured the conceptual difference between them. $K_{I}$ is a measure of the rate of electron diffusion, while $K_{E}$ is a measure of their average energy.

The densities $n_{h}$ and $n_{x}$ are the maximum densities of the electron and scattering particle distributions, which are assumed to be separable functions of plasma radius $r$ and plasma length $z$. The effective area $A_{R}$ and effective length $L_{h x}$ are the respective integrals of these normalized 
densities. In a typical quadrupole mirror field, the plasma cross-section is approximately elliptical, so the radius $r$ is simply that of the equivalent circle. The spatial variation of electron energy is neglected, as is the redistribution of the flux lines by the finite beta of the plasma.

Usually the radial density function is approximated by a parabola. In that case the area integral $A_{R}$ is

$$
A_{R}=\int_{0}^{R}\left[1-\left(\frac{r}{R}\right)^{2}\right]^{2} 2 \pi r d r=\frac{1}{3} \pi R^{2}
$$

where $n^{2}$ weighting is used, and where $R$ is the outer radius of the plasma. There is greater variety among the effective length integrals, which have the form:

$$
L_{h x}=\int_{Z_{1}}^{z_{2}} f(z) d(z)
$$

Details of the calculations of effective length $L_{h x}$ are given in Appendix $A$.

Formula (4.1) for the scattered power is true for both relativistic and nonrelativistic electrons, because the momentum $p_{h}$ and velocity $v_{h}$ are based on the Rutherford scattering formula for a single collision. For a small angle collision, the angle of deflection varies as $\left(p_{h} v_{h}\right)^{-1}$ even for relativistic particles. 8 In a plasma, the overall scattering per unit path length is a diffusion process involving many small angle collisions; therefore, it varies with the density $n$ and the square of the angle $n_{x}\left(p_{h} v_{h}\right)^{-2}$. In order to find the rate of scattering per unit time, one must multiply by the velocity $v_{h}$ to obtain the factors given in Eq. (4.1). Thus we have written Eq. (4.1) in a form which is valid for both relativistic and nonrelativistic electrons.

In the MFTF axicell design the electron kinetic energy is comparable to the rest energy $m_{0} c^{2}=511 \mathrm{keV}$, corresponding to $\gamma=2.1,2$ However, it is convenient to use nonrelativistic formulas to connect MFTF plasma physics with previous work. Let us find the ratio between the general relativistic formula and its nonrelativistic approximation. We do this by rewriting the energy-dependent factor of Eq. (4.1) as follows:

$$
\frac{k \bar{E}_{h}}{p_{h}^{2} v_{h}}=\frac{(\gamma-1) m_{0} c^{2}}{\gamma^{2} m_{0}^{2} \beta^{3} c^{3}}=\frac{\gamma(\gamma-1)}{m_{0} c\left(\gamma^{2}-1\right)^{3 / 2}} \text {. }
$$


Now substitute these same relativistic parameters into the nonrelativistic equivalent of Eq. (4.5) for the same quantity:

$$
\frac{1}{2\left[2 m_{0}\left(k E_{h}\right)\right]^{1 / 2}}=\frac{1}{2^{3 / 2} m_{0} c(\gamma-1)^{1 / 2}}
$$

From Eqs. (4.5) and (4.6), we find the ratio of the relativistic power expression $P_{S}$ to its non-relativistic approximation:

$$
\frac{P_{s}^{h x}}{P_{s}(N R)}=2^{3 / 2} \gamma\left(\frac{\gamma-1}{\gamma^{2}-1}\right)^{3 / 2}=\gamma\left(\frac{2}{\gamma+1}\right)^{3 / 2}
$$

This ratio is a convenient correction factor which converts the nonrelativistic approximation $\mathrm{P}_{S}(\mathrm{NR})$ into a more exact expression for the scattering. It approaches unity as $\gamma \rightarrow 1$, as it should, but for the extreme relativistic case it approaches $2^{3 / 2} / \gamma^{1 / 2}$, corresponding to a decrease in the scattering for high relativistic electron masses. This increased rigidity agrees with one's intuition.

In the range $1 \leq \gamma \leq 6$ (corresponding to electron kinetic energy of less than $2.5 \mathrm{MeV}$ ), one finds that the relativistic correction for scattering is less than 10\% everywhere, as shown in Fig. 8. Therefore, it need only be included for the most accurate work, and can safely be ignored for everyday estimates.

We now proceed to consider the nonrelativistic version of the scattered power Eq. (4.1). In this case, it is convenient to express $p_{h}$ and $v_{h}$ as functions of $E_{h}$. When this is done, Eq. (4.1) becomes:

$$
P_{S}(N R)=\frac{2 \pi e^{4} \ln \Lambda(\phi-G)}{\left(2 m_{0} k E_{h}\right)^{1 / 2}} \frac{K_{I} K_{E}}{\log _{10} R_{E}} n_{h} n_{x} A_{R} L_{h x}
$$

Next compare this with the other useful well-known equation for the scattered power:

$$
P_{s}^{h x}=\frac{n_{h} n_{x}}{(n \tau)_{x}} K_{I}\left(K_{E} k E_{h}\right) A_{R} L_{h x}
$$

where we have again inserted our normalizing factors $K_{I}$ and $K_{E}[E q .(4.1)]$, and where $\tau$ is the characteristic time constant for scattering. Let us solve Eq. (4.8) and (4.9) for $(n \tau)$ : 


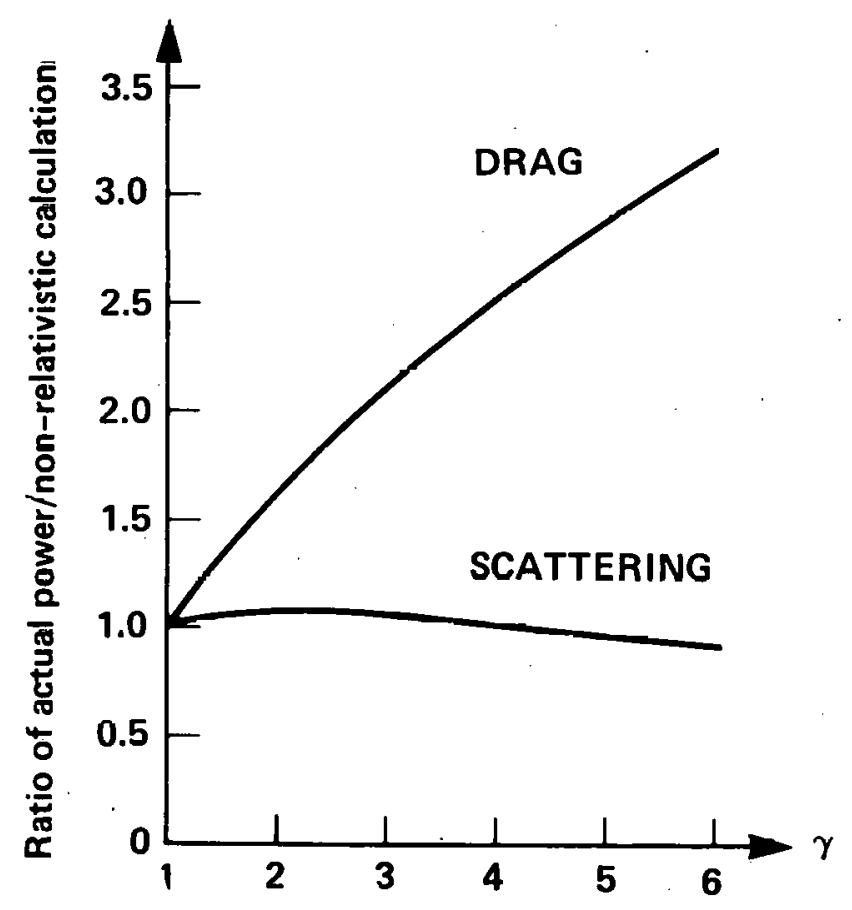

Figure 8. Relativiștic corrections to the power equations for scattering losses and drag losses. 


$$
(n \tau)_{x}=\frac{\left[2 m_{0}\left(k E_{h}\right)^{3}\right]^{1 / 2} \log _{10} R_{E}}{2 \pi e^{4} \ln \Lambda(\phi-G)}
$$
time ${ }^{9}$

The equation for $(n \tau)_{x}$ is closely related to $\tau_{D}$, the $90^{\circ}$ scattering

$$
(n \tau)_{X}=\left(2 \log _{10} R_{E}\right)\left(n \tau_{D}\right) .
$$

The multiplying factor is a fit to detailed Fokker-Planck computer calculations of collisional losses of light ions. ${ }^{10}$ Numerically Eq. $(4.10)$ is:

$$
(\mathrm{n \tau})_{\times}\left(\mathrm{cm}^{-3} \mathrm{~s}^{-1}\right)=8.2 \times 10^{9} \frac{\mathrm{E}_{\mathrm{h}}^{3 / 2} \log _{10} \mathrm{R}_{\mathrm{E}}}{\ln \Lambda(\phi-G)}
$$

where the hot electron energy $E_{h}$ is in keV.

For plasmas whose density $n \sim 10^{12}$ and temperature $T \sim 10 \mathrm{keV}$, the substitution $\ln \Lambda=20$ is a good approximation. ${ }^{9}$ When the hot electrons scatter off themselves, ${ }^{9} \phi-G \approx 0.7$. When they scatter off cold electrons or ions, ${ }^{9}$ then $\phi-G \simeq 1.0$. For such plasmas, Eq. (4.12) gives us:

$$
\begin{aligned}
& (n \tau)_{h}=5.8 \times 10^{8} E_{h}^{3 / 2}\left(\log _{10} R_{E}\right) \\
& (n \tau)_{x}=4.1 \times 10^{8} E_{h}^{3 / 2}\left(\log _{10} R_{E}\right)
\end{aligned}
$$

for the self-scattering and external scattering processes, respectively.

Suppose we consider a single electron group of average energy $E_{h}$ which is neutralized by an equal density of 'ions; then one can obtain an overall $(n \tau)_{s h}$ :

$$
(n \tau)_{s h}=\left(\frac{1}{n \tau_{h}}+\frac{1}{n \tau_{x}}\right)^{-1}=2.4 \times 10^{8} E_{h}^{3 / 2}\left(\log _{10} R_{E}\right) \text {. }
$$

Equation (4.15) is already widely used. ${ }^{1,12}$ This derivation demonstrates that it is consistent with Eq. (4.1) in the nonrelativistic approximation. Now consider the scattering of hot electrons by a separate population of "慗arm" electrons whose average energy is much less than $E_{e h}$. The warm electrons will also be neutralized by an equal density of ions, in addition to the ions which neutralize the hot electron population. In this case Eq. (4.14) 
applies to both of the scattering populations, and one obtains a time constant for this interaction $(n \tau)_{s x}$

$$
(n \tau)_{S X}=2.05 \times 10^{8} \bar{E}_{h}^{3 / 2}\left(\log _{10} R_{E}\right)
$$

The relative importance of Eq. (4.15) and Eq. (4.16) depends on the density ratios of the two populations, integrated over the magnetic well.

These formulas could be improved even more, but at the expense of considerable effort. They use an average electron energy, rather than integrating over a distribution. In the self-scattering case, both electrons are relativistic particles, but our argument was based on only one particle being relativistic. We have avoided the labor of making the appropriate transformations to find this correction, since it is likely to be no greater than the errors introduced by using the average electron energy.

\section{FOKKER-PLANCK SCATTERING RESULTS}

The previously-discussed scattering results will now be compared with some recent bounce-averaged Fokker-Planck code calculations of $r f$-heated electron distributions. 13 The calculations assume that a symmetrical magnetic well is initially filled with a Maxwellian plasma of electrons of specified low density and low temperature. For specified rf electric fields, the code calculates the buildup of a hot electron population (assuming that ions appear to neutralize their charges). One finds that the final density of hot electrons is at least an order of magnitude greater than the stream density. Therefore, we can use the code results to compare with the self-scattering Eq. (4.15), because the stream population density is low enough to neglect the scattering Eq. (4.16) and the electron-electron drag (to be discussed in the next section). The code is nonrelativistic, but we have already noted that the relativistic correction to the scattering is small (see Fig. 8). The code neglects other small effects, such as synchrotron radiation and bremsstrahlung, which should only improve the comparison with the scattering equation. 
Six of the code runs selected for the comparison used various versions of the MFTF anchor cell geometry; there were three TMX-Upgrade problems, which have a similar geometry.

One of the code outputs for each problem was the heating power per unit area:

$$
\left(\frac{d P}{d A}\right)_{C}=\frac{P s}{A_{R}}
$$

which is related to our analys is by the substitution of Eq. (4.9) for the power $P_{s}$. The value of $n \tau$ is the self-scattering formula (4.15). With these substitutions, Eq. (5.1) is solved for the product of the current and voltage constants

$$
K_{E} K_{I}=\frac{2.4 \times 10^{8}}{k} \frac{E_{h}^{1 / 2} \log _{10} R_{M}}{n_{h}^{2} L_{z}}\left(\frac{d P}{d A}\right)_{C}
$$

where we have set $R_{E}=R_{M}$ because there is no potential profile in this Fokker-Planck code [see Eq. (4.2)]. All of the quantities on the right hand side of Eq. (5.2) are available from the input or the output of each code problem.

In addition, the code output tells us the loss current per unit area $(d I / d A)_{C}$. Then the average loss energy $E_{L}$ per scattered electron (in $\mathrm{KeV}$ units) is

$$
E_{L}=\left(\frac{d P}{d A}\right)_{C} /\left(\frac{d I}{d A}\right)_{C}
$$

where the numerator is in $\mathrm{kW} / \mathrm{cm}^{2}$ and the denominater is in $\mathrm{A} / \mathrm{cm}^{2}$. But we have already pointed out that the code output tells us the average trapped electron energy $E_{h}$. Thus, we find the voltage constant $K_{E}$ :

$$
K_{E}=E_{L} / E_{h} \text {. }
$$

By combining this result with Eq. (5.2), we also find the current constant $K_{I}$. When this procedure was carried out for all nine code problems, we found that $K_{E}$ and $K_{I}$ vary from problem to problem over several orders of magnitude. However, the product $K_{E} K_{I}$ is much better behaved, as is shown in Fig. 9. Fortunately, it is just this product which is used in the power equations ([see Eq. (4.1) or (4.8)]. 


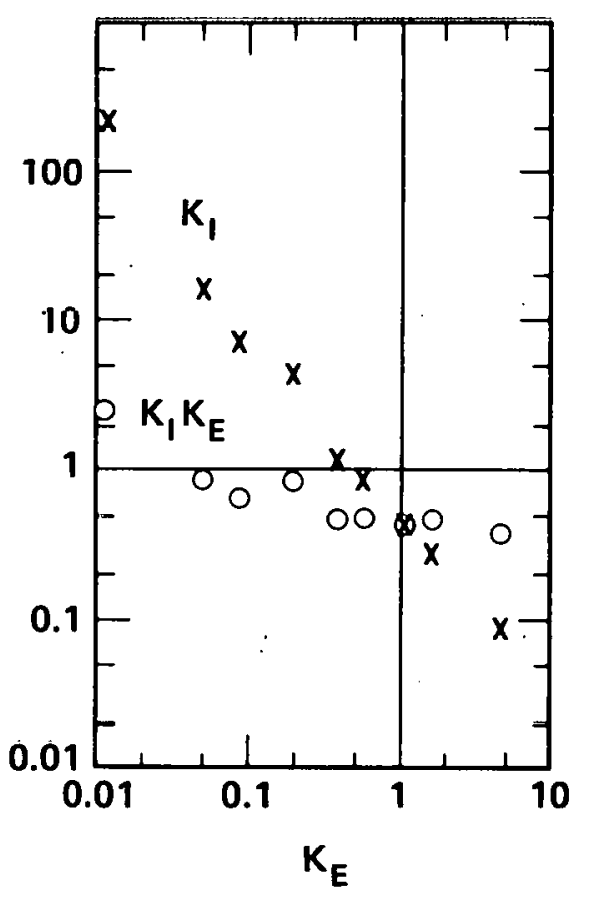

Figure 9. Variation of current and energy constants for nine Fokker Planck code runs as calculated from Eqs. (5.2) and (5.4). 


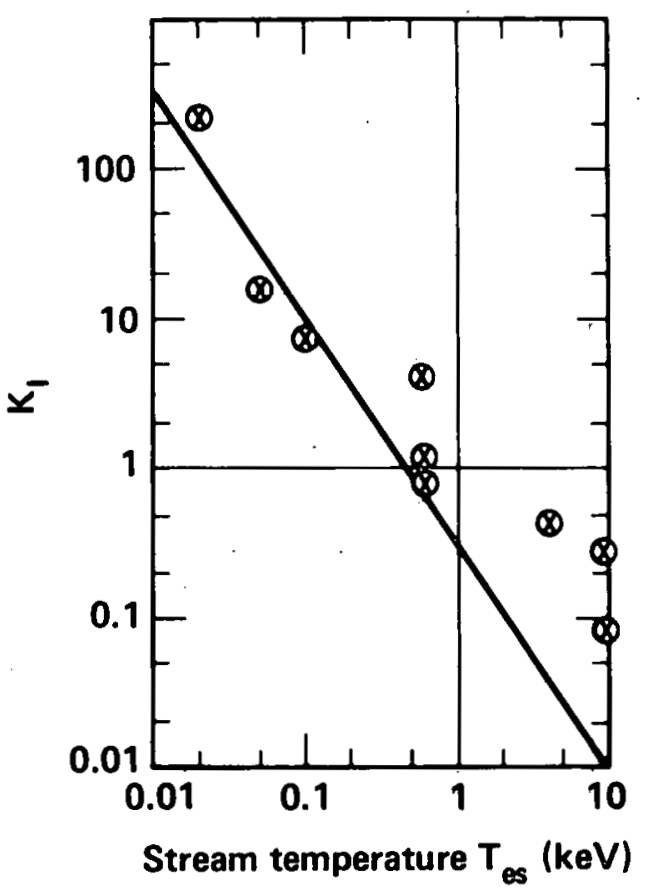

Figure 10. Comparison of the current constant $K_{I}$ with the stream temperature Tes, using Eq. (5.5) with $n_{s}=2 \times 10^{11}$. 

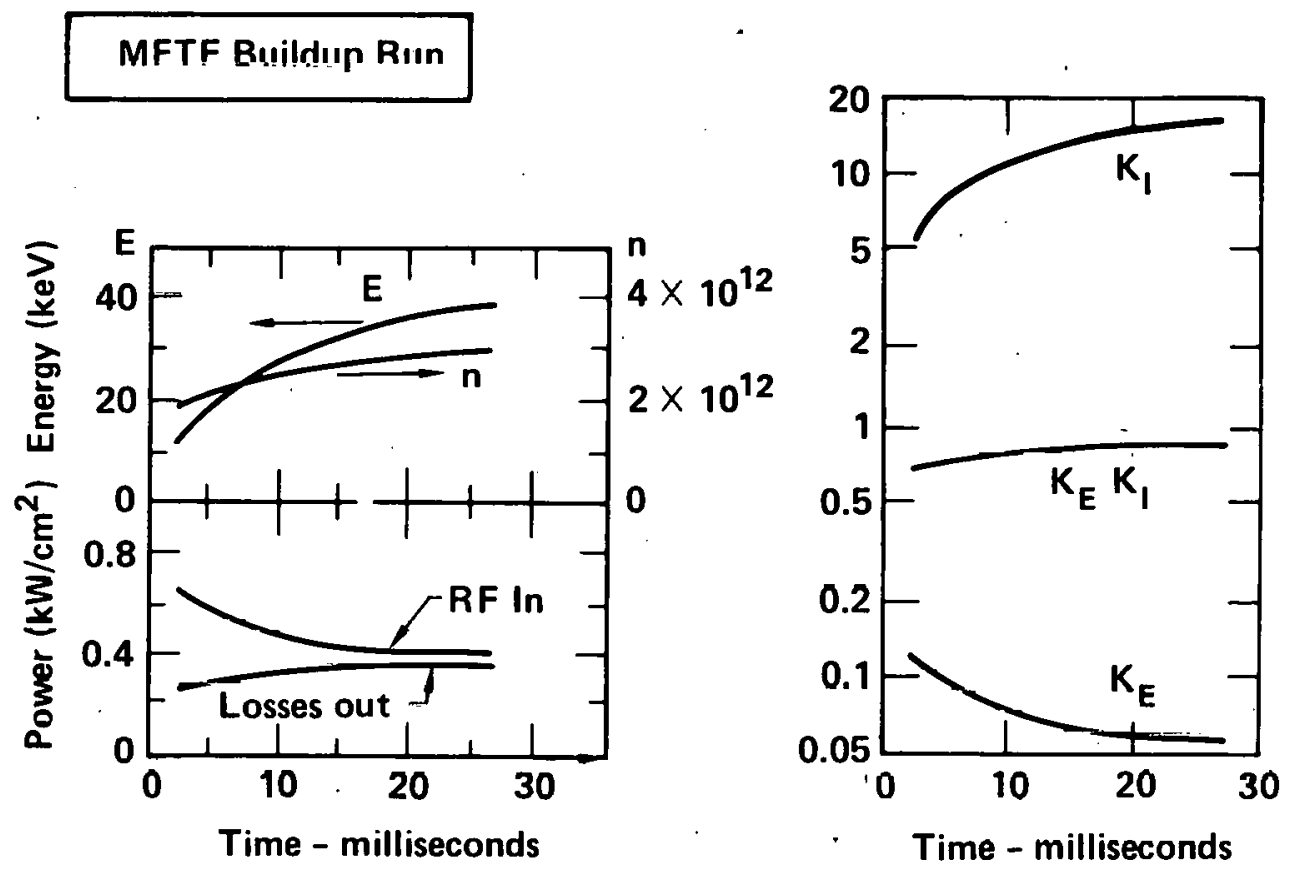

Figure 11. Time dependencc of electron density, average energy, rf power, losses, current constant $K_{I}$, energy constant $K_{E}$, and the product $K_{I} K_{E}$ plotted for one MFTF-related problem on the Fokker-Planck code. 
In an independent study of TMX-Upgrade parameters ${ }^{14}$ Grubb and Stallard used this same code to study the parameter $K_{I}$. They found that it is a strong function of the stream temperature $T_{\text {es }}$ which is assumed for each problem. From their code runs, they deduced the following fit:

$$
K_{I}=\left(7 \times 10^{-7}\right) \frac{\left(n_{s R}\right)^{1 / 2}}{\left(k_{\text {es }}\right)^{3 / 2}}
$$

where $n_{S R}$ is the stream density evaluated at the resonance. In Fig. 10, this $f i t$ is compared to the code runs used to calculate Fig. 9. One finds the same general trend of $K_{I}$ with stream temperatures.

Figure 11 shows the changes in $K_{E}$ and $K_{I}$ during a single "buildup" code run for MFTF. One sees that these coefficients evolve as the electron distribution function evolves. For this plot we used the loss power in Eq. (5.2) and (5.3), not the rf input power, which is greater than the loss power during the buildup. As equilibrium is approached, these two powers converge; the equilibrium values are used for the runs plotted in Figs. 9 and 10.

On the basis of these code calculations, the best average value of $K_{E} K_{I}$ is about one half--a value which fits the results best at the high stream temperatures which are predicted for MFTF. We shall use this value in later sections of this report.

\section{ELECTRON-ELECTRON DRAG}

In addition to scattering losses, hot electrons can lose energy to other populations by means of collisional drag. We start from the "equipartition time" $\tau_{e q}$ of a hot electron in a population $n_{x}$ of other ions or electrons. ${ }^{9}$

$$
\left(n_{x} \tau_{e q}\right) \equiv(n \tau)_{d x}=\frac{3}{8} \frac{m_{0} m_{x}}{(2 \pi)^{1 / 2} e^{4} \ln \Lambda}\left(\frac{k T_{h}}{m_{0}}+\frac{k T_{x}}{m_{x}}\right)^{3 / 2} .
$$

From Eq. (6.1) one can immediately infer that the drag time constant is much shorter for the electron-electron drag interaction than for the electron-ion case. The physical reason for this is simple; fast particles lose energy much faster by collisions with equal mass particles than by colliding with heavy particles. Consequently, we will consider only electron-electron drag between 
the hot electron population $n_{h}$ and a low temperature electron plasma, which may be either the warm electrons or the passing electrons, as described in Sec. 2 and Figs. 3 to 5 .

For the case of electron-electron drag, Eq. (6.1) becomes:

$$
(n \tau)_{d x}=\frac{1}{4 e^{4} \ln \Lambda}\left(\frac{m_{0}}{3 \pi}\right)^{1 / 2} k\left(\bar{E}_{h}+\bar{E}_{x}\right)^{3 / 2}
$$

where we have put $E=(3 / 2)$ kT. Strictly speaking, Eqs. (6.1) and 6.2) were derived only for Maxwellian velocity distributions but we shall assume that they are approximately correct for more general cases. Numerically, one obtains:

$$
(n \tau)_{d x}=8.35 \times 10^{7} \bar{E}_{h}^{3 / 2}\left(1+E_{x} / E_{h}\right)^{3 / 2}
$$

where $E_{h}$ is in keV, comparable to Eqs. (4.15) and (4.16). The overall drag power loss is similar to Eq. (4.9):

$$
P_{d}(N R)=\frac{n_{h} n_{x}}{(n \tau)_{d x}} k\left(\bar{E}_{h}-\bar{E}_{x}\right) A_{R} L_{h x} \text {. }
$$

When one substitutes Eq. (6.2), this power becomes:

$$
P_{A}(N R)=\frac{4 \sqrt{3 \pi} e^{4} \ln \Lambda}{\left(1110 k E_{h}\right)^{1 / 2}} \frac{1-E_{x} / E_{h}}{\left(1+E_{x} / E_{h}\right)^{3 / 2}} n_{h} n_{x} A_{R} L_{h z} \text {. }
$$

Up to this point, all of the drag equations are nonrelativistic; in order to correct them, we must first write Eq. (6.5) in terms of the hot electron velocity $v_{h}$ :

$$
P_{d}^{h x}=\frac{4 \sqrt{6 \pi} e^{4} \ln \Lambda}{m_{0} v_{h}} \frac{1-E_{x} / E_{h}}{\left(1+E_{x} / E_{h}\right)^{3 / 2}} n_{h} n_{x} A_{R} L_{h x} .
$$

The velocity term $1 / v_{h}$ is consistent with the Rutherford scattering formula for a single collision. Instead of the deflection angle (as in Eq. 4.1), we are now concerned with the energy loss per collision, 8 which varies as $\left(v_{h}\right)^{-2}$. In this case, the energy loss per unit length is directly proportional to the number of collisions times the energy loss per collision 
$n_{x}\left(v_{h}\right)^{-2}$. Then the rate per unit time is found by multiplying by the velocity $v_{h}$ in order to obtain the factors given in Eq. (6.6). The electron rest mass $m_{0}$ is not involved, because it is the mass of the "warm" electron, which is assumed to be nonrelativistic.

This argument ignores relativistic adjustments of the correction term for the energy ratio $E_{x} / E_{h}$. Such corrections, however, would be complicated and laborious because they involve repeating Spitzer's calculations with relativistic Maxwellian distributions. In practical cases, we always expect $E_{x} \ll m_{0} c^{2}$, so that the terms in $E_{x} / E_{h}$ would be small when $E_{h} \simeq m_{0} c^{2}$, and that is the only case for which the relativistic correction is significant.

Thus, the relativistic correction for the drag power involves only the velocity term $v_{h}^{-1}$. If one carries out a derivation similar to the procedure used in Eq. (4.5) to (4.7), one obtains the ratio:

$$
\text { - } \frac{p_{d}^{h x}}{p_{d}(N R)}=\gamma\left(\frac{2}{\gamma+T}\right)^{1 / 2}
$$

As shown in Fig. 8, this is a significant correction for all electron energies greater than $\simeq 100 \mathrm{keV}$. As one can see from $\mathrm{Eq} \cdot(6.6)$, when $v_{h} \rightarrow c$ the actual drag power becomes independent of hot electron energy. Physically, this means that the cold electrons (which absorb the energy) are responding only to the coulomb forces of the electron charge (which is a relativistic invariant), and not to the mass of the fast electron.

Finally, it should be emphasized that unlike the scattered power, the drag power is not lost from the system. It is simply transferred from the hot electrons to the cooler electron populations, and is an important term in the internal energy balance.

\section{RADIATION LOSSES}

For densities $10^{12}$ to $10^{13}$, temperatures of 10 to $1000 \mathrm{keV}$, and magnetic fields of 0.1 to $10 \mathrm{~T}$ the dominant radiation loss is magnetic bremsstrahlung--usually called synchrotron radiation. Ordinary electron-ion and electron-electron bremsstrahlung are negligible power losses, ${ }^{15}$ even though they are important for diagnostics purposes. The equation for the synchrotron radiation power loss $P_{\text {sy }}$ is: ${ }^{8}$ 


$$
P_{s y}^{h}=\frac{2}{3} r_{0}^{2} C B^{2}\left(\gamma^{2}-1\right) n_{h} A_{R}^{\prime} L_{h}^{\prime}
$$

where $r_{0}$ is the classical electrical radius. We neglect reabsorption effects, which are small for the plasmas of interest to us. ${ }^{16}$ The area integral $A_{R}^{\prime}$ and the length integral $L_{h}^{\prime}$ are discussed in Appendix $A$.

Because synchrotron losses have quite a different functional dependence on $n_{h}, B$, and average energy $\left(E_{h}\right.$ or $\left.\gamma\right)$, the $(n \tau)_{\text {sy }}$ function makes an interesting contrast with the previous ones. A comparison of Eq. (4.9) and (7.1) shows us how to define it:

$$
\frac{n_{h}^{2}}{(n \tau)_{s y}}\left(k E_{h}\right)=\frac{2}{3} r_{0}^{2} c B^{2}\left(\gamma^{2}-1\right) n_{h} \text {. }
$$

where $K_{E}=1$, and where we have chosen to make our estimate at a specific point (the midplane) with given values of $B_{0}$ and $n_{h}$. Now $k E_{h}$ is just the kinetic energy, so we can combine Eq. (2.2) with Eq. (7.2), and solve,

$$
(n \tau)_{s y}=\frac{3}{2} \frac{m_{0} c}{r_{0}^{2}} \frac{n_{h}}{B_{0}^{2}} \frac{1}{\gamma+1}=\frac{5.16 n_{h}}{(\gamma+1)\left(B_{T}\right)^{2}}
$$

where $B_{T}$ is the magnetic field in teslas. Comparing Eq. (7.3) with similar equations for scattering [(Eqs. (4.13) and (4.14)] and drag [Eq. (6.3)], we see that the synchrotron radiation expression $(n \tau)_{\text {sy }}$ is not just a function of electron energy, but it also depends on the electron density and the magnetic field. This different functional dependence also implies different area and length integrals, as described in Appendix A.

\section{SUMMARY OF HOT ELECTRON LOSSES}

Let us now rewrite our power loss results in a form which is convenient for further use. The total power loss $P_{T}^{h}$ of the hot electrons is the sum of several individual terms

$$
P_{T}^{h}=P_{s}^{h h}+P_{s}^{h p}+P_{s}^{h w}+P_{d}^{h p}+P_{d}^{h w}+P_{s y}^{h}
$$

where the first three terms are scattering losses, the next two are drag losses, and the last is the synchrotron term. 
The scattering loss terms $P_{s}^{h x}$ are all of the form:

$$
P_{S}^{h x}=n_{h}\left(k E_{h}\right)\left(K_{E} K_{I}\right) \frac{n_{e x}}{(n \tau)_{s x}} A_{R} L_{h x} \gamma\left(\frac{2}{\gamma+1}\right)^{3 / 2}
$$

where $x$ represents either hot electrons $(h)$, warm electrons $(w)$, or passing electrons $(p) . \quad(n \tau)_{s x}$ is the usual nonrelativistic $n \tau$ which includes scattering from both the electrons of density $n_{x}$ and an equal number of charge-neutralizing ions. $(n \tau)_{s h}$ comes from Eq. $(4.15) ;(n \tau)_{S W}$ and $(n \tau)_{s p}$ are both given by Eq. (4.16); and the product $K_{E} K_{I}$ is one half, as discussed in Sec. 5.

The drag loss terms $\mathrm{p}_{\mathrm{d}}^{\mathrm{hx}}$ are given by:

$$
P_{d}^{h x}=n_{h} k\left(E_{h}-E_{x}\right) \frac{n_{x}}{(n \tau)_{d x}} A_{R} L_{h x} \gamma\left(\frac{2}{\gamma+1}\right)^{1 / 2}
$$

where $(n \tau)_{d x}$ is the nonrelativistic Eq. (6.3).

The synchrotron losses $P_{x y}^{h}$ are given in the previous section.

\section{HOT ELECTRON POWER LOSS FOR A SIMPLE MODEL}

We wish to estimate the required ECRH heating power at point (b) in the MFTF anchor region (see Fig. 5), and compare it with the TMX-Upgrade anchor region. For this purpose we use the simple linear density profiles shown in Fig. 12 as an approximate representation of the expected overlap of electron populations. 2,12 Table 1 presents the resultant area and length ratios as calculated from Appendix A, along with other common parameters chosen to keep the comparison between the two experiments as simple as possible.

All of the other required parameters for the model calculations are different for the two experiments; these are set out in Table 2. The minimum field $B_{0}$, the vacuum field $B_{V}$, and the hot electron average energy $E_{h}$ are consistent with the equations:

$$
\begin{gathered}
B_{0}=B_{V} \sqrt{1-\beta} \\
B=8 \pi n_{h}\left(k E_{h}\right) / B_{V}^{2} .
\end{gathered}
$$




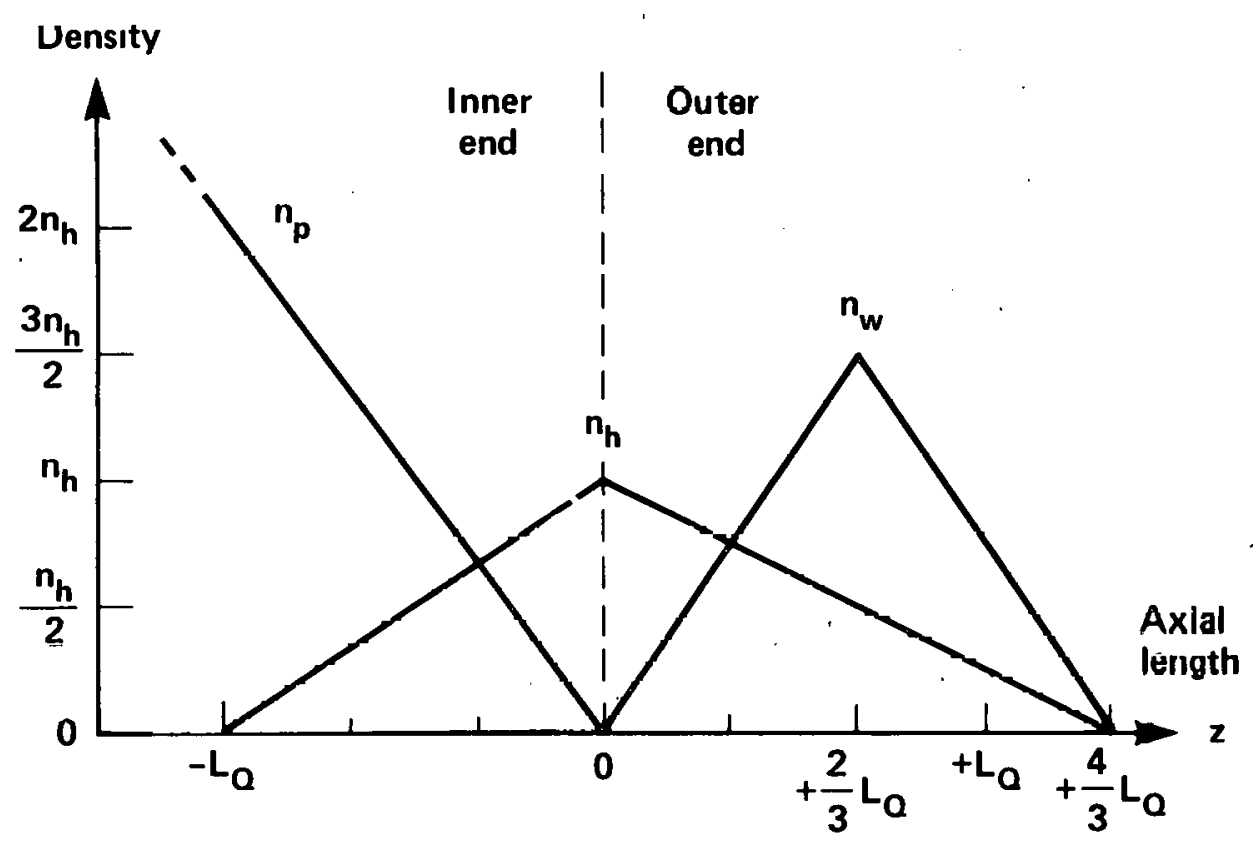

Figure 12. Approximate linear model of electron density distributions in a quadratic magnetic well--for use in estimating electron power losses, based roughly on the profiles in Fig. 5. 
Table 1. Common parameters of the comparison.

Common Parameters of Model

Hot electron density

Equivalent mirror ratio

Scattering power correction factor

$$
\begin{aligned}
& n_{h}=4 \times 10^{12} \mathrm{~cm}^{-3} \\
& R_{E}=4.75 \\
& K_{I} K_{E}=0.5
\end{aligned}
$$

Area and Length Ratios*

$\begin{array}{lllll}\text { Inner side } & h h & 1 / 3 & & 0.3068 \\ & h p & 1 / 3 & \\ & h & 1 / 2 & 0.1320 \\ \text { Outer side } & h h & 1 / 3 & 0.5833 \\ & h w & 1 / 3 & 0.3895 \\ & h & 1 / 2 & 0.3801 \\ & h & & 0.7292\end{array}$

${ }^{*} L_{Q}$ is the characteristic length of the quadratic well as given in Table 2. 
Table 2. Characteristic parameters of the anchor region of two experiments.

\begin{tabular}{lcc}
\hline Parameter & TMX-Upgrade & MFTF \\
\hline & & \\
$\mathrm{L}_{Q}(\mathrm{~cm})$ & 15 & 150 \\
$\mathrm{R}_{0}(\mathrm{~cm})$ & 15 & 30 \\
$\mathrm{~B}_{V}(\mathrm{kG})$ & 5 & 10 \\
$B$ & 0.32 & 0.55 \\
$\mathrm{~B}_{0}(\mathrm{kG})$ & 4.1 & 5.7 \\
& & \\
Electron energies & & 344 \\
$E_{h}$ (keV) & 50 & 75 \\
$E_{W}$ (keV) & 4.35 & 13.5 \\
$E_{p}$ (keV) & 0.90 & \\
\hline
\end{tabular}


Using the parameters in Tables 1 and 2 , we obtain the results set out in Table 3 for the various power terms of Eq. (8.1). The MFTF anchor model requires more power than the TMX-Upgrade case because of its larger plasma size, which overshadows the temperature effect. The total power loss is essentially the ECRH heating requirement for the microwaves which heat the hot electron component, where we have neglected the energy of the "feed current" of electrons. This approximation is best for cases where $K_{1}$ is small (and $n \tau$ is large), corresponding to the high stream temperature of MFTF.

If we scale the MFTF anchor experiment by holding $\beta$ constant (at $\beta=0.55)$ in Eq. (9.2), then we can find how the loss power varies with hot electron energy $E_{h}$. The results are shown in $\mathrm{Fig} .13$, where the loss powers are plotted versus hot electron energy; and show a strong decrease with increasing energy, because the density varies inversely with energy at constant beta. The synchrotron power follows a different scaling law, but it is only a small correction in this range.

Figure 13 illustrates the desirability of having two $200 \mathrm{~kW}$ gyrotron tubes to heat the hot MFTF electrons. If we assume $75 \%$ efficiency for the energy transfer from the gyrotron to the electrons, then two tubes will balance $300 \mathrm{~kW}$ of losses, corresponding to a hot electron temperature of $260 \mathrm{keV}$ or more. One tube would only balance $150 \mathrm{~kW}$, corresponding to the higher temperature of $350 \mathrm{keV}$ or more.

\section{POWER ESTIMATES FOR THE WARM.ELECTRONS}

Here we make an approximate estimate of the ECRH heating power $P_{W}$ for the warm electrons at point (a) (see Fig. 5). We neglect scattering losses, and assume that the dominant energy requirement arises from cold electrons produced by ionization of the two neutral beam sources--the sloshing ion source and the high energy pump beam (HEPB). Then the "strong ECRH" power equation $^{2}$ is:

$$
P_{w}=J_{c e}\left(\frac{R_{a b} \delta \phi_{a}}{T-R_{a b}}+\frac{T_{w}}{R_{a b}}\right) .
$$

where $J_{c e}$ is the cold electron current, $R_{a b}$ is the mirror ratio between $L_{n}=(2 / 3) L_{Q}$ and the midplane (see Fig. 12) and $\delta \phi_{a}$ is the potential difference between them. 
Table 3. Power losses of hot electrons in each anchor.

\begin{tabular}{|c|c|c|c|}
\hline $\begin{array}{c}\text { Symbol } \\
{[\mathrm{Eq} \cdot(8.1)]}\end{array}$ & Type of power loss & TMX-U model & MFTF model \\
\hline$P_{S}^{h h}$ & Scattering (self) & 13.91 & 44.9 \\
\hline$P_{s}^{h p}$ & Scattering (on $n_{p}$ ) & 3.11 & 10.0 \\
\hline$P_{S}^{h w}$ & Scattering (on $n_{w}$ ) & 8.96 & 28.9 \\
\hline$p_{d}^{h p}$ & $\operatorname{Drag}\left(\right.$ on $\left.n_{p}\right)$ & 5.19 & 20.1 \\
\hline$p_{d}^{h w}$ & Drag (on $n_{w}$ ) & 12.59 & 37.3 \\
\hline$p_{\text {sy }}^{h l}$ & Synchrotron & 0.07 & 14.2 \\
\hline$P_{T}^{h}$. & Total power loss (kW) & 43.83 & 155.4 \\
\hline
\end{tabular}




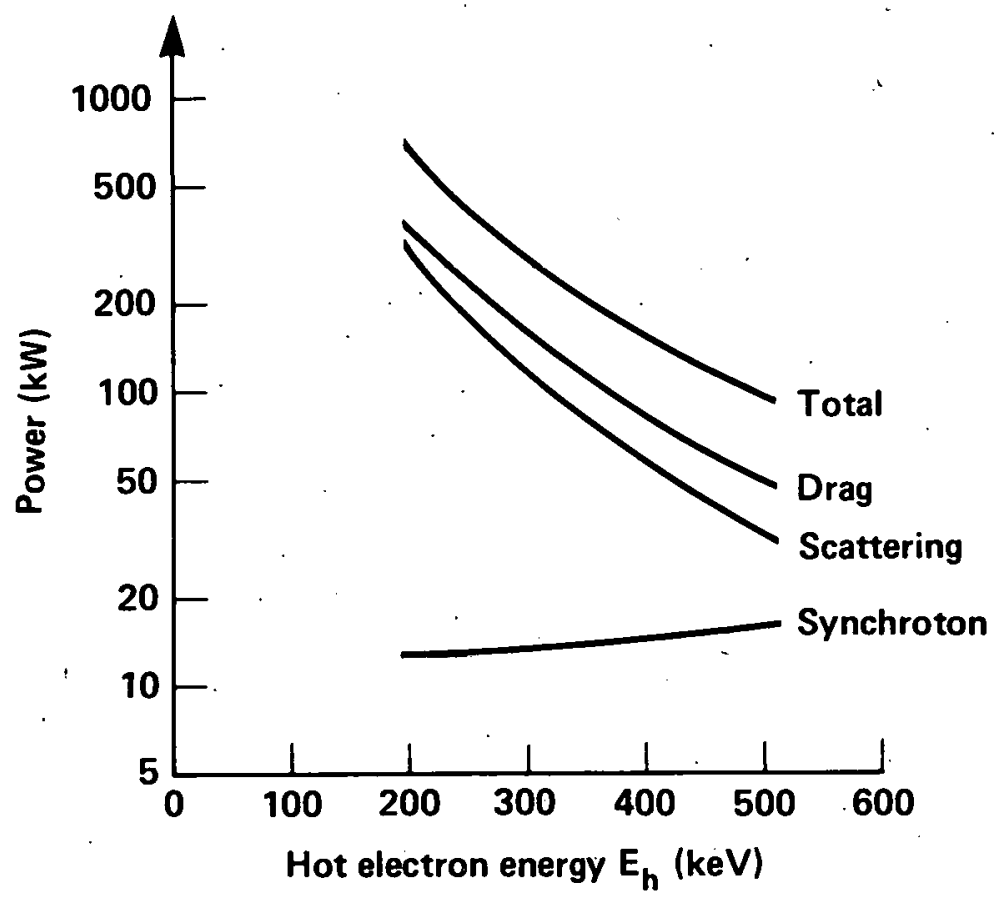

Figure 13. Hot electron loss powers versus electron energy in the MFTF anchor model calculation at constant beta. 
The cold electron current $I_{c e}$ is:

$$
I_{c e} \simeq n_{b} \sigma_{B} J_{B} A_{R}^{\prime}(\Delta Z)_{B}\left(1+f_{H E P B}\right)
$$

where the sloshing ion neutral beam parameters are $J_{B}$ (current density), $\Delta Z_{B}$ (beam width), and $\sigma_{B}$ (beam ionization cross-section). The parameter $n_{b}$ is the total electron density at $Z=L_{N}=(2 / 3) L_{Q}$; for a typical. sloshing ion beam width $\Delta Z_{B}=25 \mathrm{~cm}$, we use a constant value of $n_{b} \cdot f_{H E P B}$ is the fraction of sloshing ions that are pumped on the warm electron side of the thermal barrier by the HEPB. In this approximate estimate we assume that $J_{B}$ is constant over the plasma cross-section; therefore, the equivalent area $A_{R}$ is given by Eq. (A.2) of Appendix A.

The neutral beam ionization cross-section $\sigma_{B}$ (ignoring corrections for the half and third energy components) is:

$$
\sigma_{B}=\sigma_{i i}+\frac{v_{w}}{v_{R}} \sigma_{e i}
$$

where $\sigma_{i j}$ is the ion-neutral ionization cross-section for the neutral beam atoms incident on the sloshing ion population, where $\sigma_{e i}$ is the electron-neutral ionization cross-section for electrons of mean energy $E_{W}$, where $v_{W}$ is the corresponding electron velocity, and $v_{B}$ is the velocity of neutral beam atoms.

The sloshing ion beam current density $J_{B}$ depends on the total beam current $I_{B}$ and the achievable tocus conditions for a plasma distance of $\simeq 10 \mathrm{~m}$. In this estimate we assume a square beam of dimensions $\Delta Z_{B}=25 \mathrm{~cm}$, which is compatible with the present neutral beam state-of-the-art. Combining all of the above equations and definitions, one finds

$$
I_{c e}=\left(\pi R^{2} n_{b} I_{B} / 2(\Delta Z)_{B}\right)\left(\sigma_{i j}+\frac{V_{w}}{V_{B}} \sigma_{e i}\right)\left(1+f_{H E P B}\right) .
$$

Fquations $(10,1)$ and $(10.4)$ are the required relations for determining the estimated power needed to heat the cold electrons introduced by ionization of the sloshing ion beam. As previously stated, some of the warm electron heating arises from drag power losses of the hot electrons. Thus the required ECRH power $P_{E C R H}(a)$ must be obtained from:

$$
P_{E C R H}(a)=P_{w}-P_{d}^{h w}
$$


where point (a) is indicated in Fig. 5 and $P_{d}^{h w}$ is explained by Eq. (8.3). In Table 4 the results of all these calculations are shown, including this drag correction, in order to obtain an estimate of the ECRH power requirement. The three MFTF examples used are compatible with the earlier constant $B$ scaling results (Fig. 13).

The results of Table 4 show the need for two $200 \mathrm{~kW}$ gyrotron power sources at point (a). Assuming $75 \%$ efficiency [as we did in the previous section for point (b)], we find that the losses can be balanced in the same temperature range as for point (b). Actually, two $200 \mathrm{~kW}$ gyrotrons are assigned to this heating location, and they have different frequencies (28 $\mathrm{GHz}$ and $35 \mathrm{GHz}$ ), as described in Sec. 3 (see also Fig. 6). The colder electrons in the distribution are more efficiently heated by the $35 \mathrm{GHz}$ waves; the hotter ones by $28 \mathrm{GHz}$. More exact details of the heating process are beyond the capabilities of this simple model, but one should expect improved heating effectivity from the combination.

\section{ECRH REQUIREMENTS FOR MFTF AXICELL DESIGN}

This report has described the considerations that led to the electron cyclotron resonance heating (ECRH) requirements specified for the MFTF axicell. Table 5 summarizes them for each anchor region. 17 The absorbed powers are compatible with high hot electron energy $\left(E_{h}>250 \mathrm{keV}\right)$. The specified.frequencies are identical to those considered here. The major difference from the TMX-Upgrade experiment is the necessity to include relativistic effects in the estimates. Such effects are particularly important for evaluating resonance frequency shifts, electron-electron drag, and synchrotron radiation.

\section{ACKNOWLEDGMENTS}

This work was assisted by numerous other individuals, especially R. Jong, K. Krause, B. G. Logan, Y. Matsuda, G. Porter, B. Stallard, and J. Yugo. 
Table 4. Parameters for MFTF anchor warm electron heating.

\begin{tabular}{|c|c|c|c|}
\hline$v_{B}=2.8 \times 10^{8} \mathrm{~cm} / \mathrm{s}$ & \multicolumn{3}{|c|}{ (80 keV neutrals) } \\
\hline$v_{w}=1.4 \times 10^{10} \mathrm{~cm} / \mathrm{s}$ & \multicolumn{3}{|c|}{ (75 keV electrons) } \\
\hline$\sigma_{i i}=1.6 \times 10^{-16} \mathrm{~cm}$ & & & \\
\hline$\sigma_{e 1}=3 \times 10^{-19} \mathrm{~cm}^{2}$ & \multicolumn{3}{|c|}{$\sigma_{B}=\cdot 1.75 \times 10^{-16} \mathrm{~cm}^{2}$} \\
\hline$I_{B}=15 \mathrm{~A}$ & \multicolumn{3}{|c|}{$\delta \phi_{a}=/ b \mathrm{kV}$} \\
\hline$(\Delta Z)_{B}=25 \mathrm{~cm}$ & \multicolumn{3}{|c|}{$k_{a b}=1.44$} \\
\hline$A_{R}=300 \pi \mathrm{cm}^{2}$ & \multicolumn{3}{|c|}{$T_{w}=2 / 3(75) \mathrm{kV}}$. \\
\hline & \multicolumn{3}{|c|}{ Three cases at $\beta_{\text {eh }}=0.55$} \\
\hline$E_{h}(k e V)$ & 204 & 344 & 511 \\
\hline$n_{b}=2 n_{h}\left(\mathrm{~cm}^{-3}\right)^{\star}$ & $13.5 \times 10^{12}$ & $8 \times 10^{12}$ & $5.4 \times 10^{12}$ \\
\hline$I_{c e}(A)$ & 2.01 & 1.19 & 0.80 \\
\hline$P_{W}(k W)$ & 559 & 331 & 223 \\
\hline$P_{d}^{h w}$ & 244 & 74 & 32 \\
\hline$P_{\text {ECRH }}$ (a) & 315 & 257 & 191 \\
\hline$P_{E C R H}$ (b) (Fig. 13) & 693 & $\underline{213}$ & 95 \\
\hline$P_{\text {ECRH }}($ Total $)$ & 1008 & 470 & 286 \\
\hline
\end{tabular}

*See Fig. 12, at $z=+\frac{2}{3} L_{Q}$ 
Table 5. ECRH power distribution for each anchor region of the MFTF axicell.

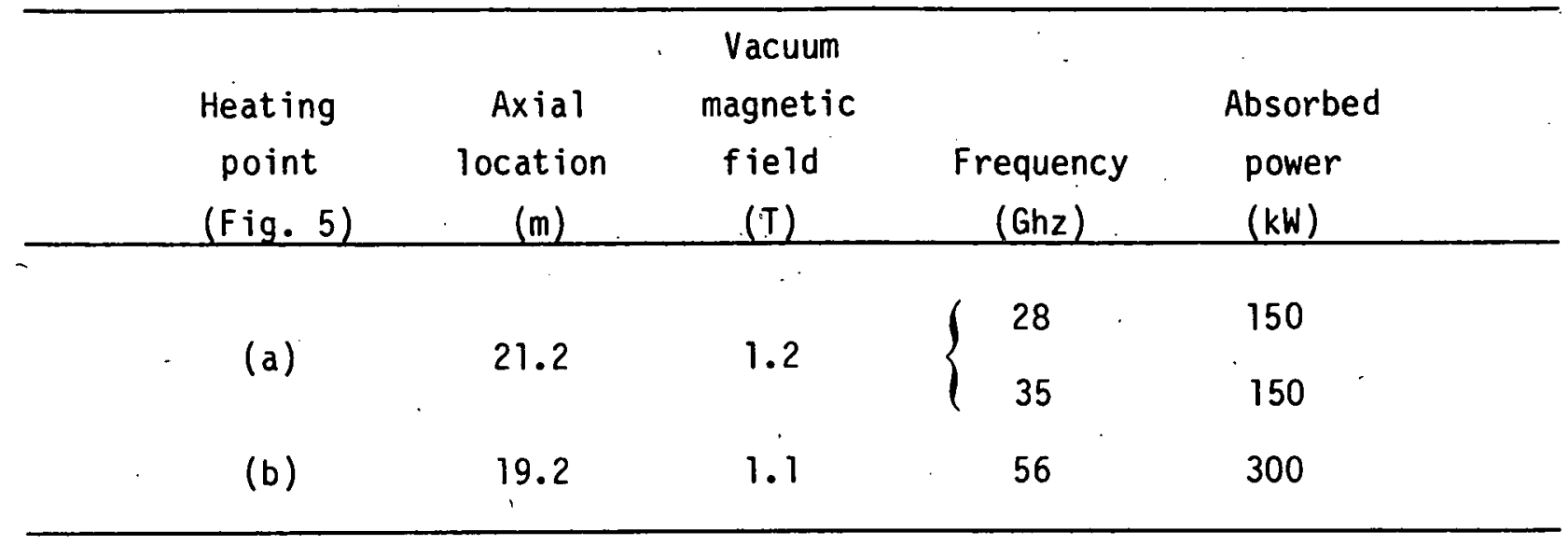




\section{REFERENCES}

1. K. I. Thomassen and V. N. Karpenko, "An Axicell Design for the End Plugs of MFTF-B," Lawrence Livermore National Laboratory, Livermore, CA, Rept. UCID-19318 (April 1982)

2. D. E. Baldwin and B. G. Logan, "Physics Basis for an Axicell Design for the End Plugs of MFTF-B," Lawrence Livermore National Laboratory, Livermore, CA, Rept. UCID-19359 (Apri1 1982).

3. Y. Matsuda and J. J. Stewart, "Fokker-Planck Calculations of Hot and Warm Electrons in a Tandem Mirror Plug," abstract submitted for the 24th Annual Meeting, Division of Plasma Physics, Nov. 1-5, 1982.

4. B. R. Stallard; Y. Matsuda, and W. M. Nevins, "rokker-HIanck Calculations of Hot-Electron Formation by Electron Cyclotron Resonance Heating (ECRH) in the TMX-Upgrade Tandem Mirror," Lawrence Livermore National Laboratory; Livermore, CA, Rept. UCRL-87674 Preprint, submitted to Nuclear Fusion (June 1982).

5. J.W. Shearer, "Doppler-Shifted Resonance Absorption by Hot Electrons," pp. 583-595 of Proceedings of the 2nd Workshop on Hot Electron Ring Physics, ORNL Report CONF-811203 (June 1982).

6. R. A. Dand1, et al., "High Beta Relativistic Electron Plasmas in Axisymmetric and Nonaxisymmetric Mirrors," Proc. 4th IAEA Conference, Vol. II, 607 (1971).

7. W. B. Ard, et a1., "Use of a Hot-Electron Target Plasma for Accumulation of Energetic Ions in Stabilized Magnetic Mirror Traps," Proc. 4th IAEA Conference, Vol. II, 619 (1971).

8. J. D. Jackson, "Classical Electrodynamics," 2nd edition, John Wiley and Sons, New York (1975).

9. Lyman Spitzer, Jr., "Physics of Fully Ionized Gases," 2nd edition, Interscience, New York (1962).

10. A. H. Futch, Jr., J. P. Holdren, J. Killeen, and A. A. Mirin, "Muiti-Species Fokker-Planck Calculations for D-T and D- ${ }^{3}$ He Mirror Reactors," Plasma Physics 14, 211-244 (1972).

11. B. G. Logan, "MFTF-B Operation with Thermal Barriers," pp. (II-31) $(1 I-115)$ of "Physics Basis for MFIF-B," Lawrence Livermore National Laboratory, Livermore, CA, Rept. UCID-18496, Pt. I (1980). 
12. B. G. Logan, "TMX Upgrade Operating Parameters with Thermal Barriers," pp. $(A-17)$ - $(A-50)$ of "TMX Upgrade Major Project Proposal," Lawrence Livermore National Laboratory, Livermore, CA, Rept. LLL-PROP-172 (1980).

13. Ref. 4, p. 10ff.

14. D. P. Grubb and B. W. Stallard, private communmication (March 1982).

15. S. K. Borokowski, N. A. Uckan, E. F. Jaeger, and T. Kammash, "Ring Power Balance," pp. 319-332 of Proc. of the Workshop on EBT Ring Physics, ORNL Rept,. CONF-791228 (1980).

16. B. A. Trubnikov, "Universal Coefficients for Synchrotron Emission from Plasma Configurations," p. 345ff of "Reviews of Plasma Physics," Vol. 7 , Consultants Bureau, New York (1979).

17. Table 3.3.7-1 of Ref. 1. 


\section{APPENDIX A. EFFECTIVE AREA AND LENGTH INTEGRALS}

As described in Sec. 4, the plasma densities are first normalized to their maximum values, and then the total scattered power is found by integrating over the radial variable $r$ and the length variable $z$. In these calculations the electron energy $E_{h}$ is assumed to be a constant. The area and length integrals [Eqs. (4.3) and (4.4)] are also applicable to the drag equations, which have the same density dependence. Other integrals, described below, are needed for the synchrotron power, which is a different function of density and magnetic field.

Consider the area integrals first. For the scattering and drag terms, we use tq. (4.3), which is repeated here,

$$
A_{R}=\int_{0}^{R}\left[1-\left(\frac{r}{R}\right)^{2}\right]^{2} 2 \pi r d r=\frac{1}{3} \pi R^{2}
$$

corresponding to a parabolic density profile. For the synchrotron case, the area integral $A_{R}^{\prime}$ is different because $P_{s y}^{h}$ varies only linearly with density $n$ :

$$
A_{R}^{\prime}=\int_{0}^{R}\left[1-\left(\frac{r}{R}\right)^{2}\right] 2 \pi r d r=\frac{1}{2} \pi R^{2} .
$$

Now consider the length integrals. Equation (4.4) for the effective length $L_{h x}$ is an integral of the form;

$$
L_{h x}=\int_{z_{1}}^{z_{2}} \frac{n_{h}(z)}{n_{h}} \frac{n_{x}(z)}{n_{x}} \frac{B_{0}}{B(z)} d z
$$

where $B_{0}$ is defined at the midplane. The field ratio $\left[B_{0} / B(z)\right]$ accounts for the variation in the cross-sectional area of the magnetic flux tube.

For the model calculations in this paper, simple approximate functions are used for the ratios in Eq. (A.3). The field protile (see Fig. 5) is approximated by a quadratic well:

$$
B(z) / B_{0}=1+\left(z / L_{Q}\right)^{2}
$$

where $z$ is measured from the midplane. Two linear density functions are defined: 


$$
F_{1}=1-\frac{Z}{L_{N}} ; F_{2}=\frac{Z}{L_{N}}
$$

When the approximate Eqs. (A.4) and (A.5) are substituted into Eq. (A.3), one obtains the following results:

$$
\begin{aligned}
& S_{11} \equiv \int_{0}^{L_{N}} F_{1}^{2} \frac{d z}{1+\left(z / L_{Q}\right)^{2}}=a_{Q}\left[\frac{1-a^{2}}{a} \tan ^{-1} \frac{1}{a}+1-\ln \left(1+\frac{1}{a^{2}}\right)\right] \\
& S_{12} \equiv \int_{0}^{L_{N}} F_{1} F_{2} \frac{d z}{1+\left(z / L_{Q}\right)^{2}}=a L_{Q}\left[a \tan ^{-1} \frac{1}{a}-1+\frac{1}{2} \ln \left(1+\frac{1}{a^{2}}\right)\right] \\
& S_{22} \equiv \int_{0}^{L_{N}} F_{2}^{2} \frac{d z}{1+\left(z / L_{Q}\right)^{2}}=a L_{Q}\left(1-a \tan ^{-1} \frac{1}{a}\right) \\
& S_{1} \equiv \int_{0}^{L_{N}} F_{1} \frac{d z}{1+\left(z / L_{Q}\right)^{2}}=L_{Q}\left[\tan ^{-1} \frac{1}{a}-\frac{1}{2} \ln \left(1+\frac{1}{a^{2}}\right)\right] \\
& S_{2} \equiv \int_{0}^{L_{N}} F_{2} \frac{d z}{1+\left(z / L_{Q}\right)^{2}}=L_{Q}\left[\frac{a}{2} \ln \left(1+\frac{1}{a^{2}}\right)\right]
\end{aligned}
$$

where the parameter $a \equiv L_{a} / L_{N}$. These integrals, or linear combinations of them, are useful to find $L_{h x}$ for particular models of the density functions.

If $F_{1}$ and $F_{2}$ were parabolic functions rather than linear, then we could also find elementary function solutions of these integrals. But they are longer equations, requiring more labor. The values of $L_{h x}$ obtained from them do not differ greatly from the linear function cases, when the same general shapes of the density functions are being modeled. Thus, we use the linear approximations given here. 
For the synchrotron power loss equation, the length integral $L_{h}^{\prime}$ is

$$
L_{h}^{\prime}=\int_{Z_{1}}^{Z_{2}} \frac{n_{h}(z)}{n_{h}}\left[\frac{B(z)}{B_{0}}\right]^{2} \frac{B_{0}}{B(z)} d z .
$$

In terms of the previous approximations, a useful integral for this parameter is

$$
S^{\prime}=\int_{0}^{L_{N}} F_{1}\left[1+\left(\frac{z}{L_{Q}}\right)^{2}\right]=\frac{L_{Q}}{2 a}\left(1+\frac{1}{6 a^{2}}\right) .
$$

The magnetic field dependence is seen to introduce a considerable difference between the integrals $s_{1}$ and $s_{1}^{\prime}$. 


\section{DISCLAIMER}

This document was prepared as an account of work sponsored by an agency of the United States Government. Neither the United States Government nor the University of California nor any of their employees, makes any warranty, express or implied, or assumes any legal liability or responsibility for the accuracy, completeness, or usefulness of any information, apparatus, product, or process disclosed, or represents that its use would not infringe privately owned rights. Reference herein to any specific commercial products, process, or service by trade name, trademark, manufacturer, or otherwise, does not necessarily constitute or imply its endorsement, recommendation, or favoring by the United States Government or the University of California. The views and opinions of authors expressed herein do not necessarily state or reflect those of the United States Government thereof, and shall not be used for advertising or product endorsement purposes.

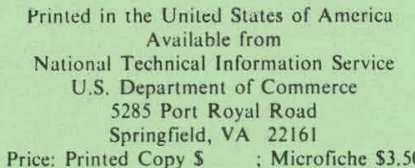

\begin{tabular}{|c|c|c|c|}
\hline Page Range & $\begin{array}{l}\text { Domestic } \\
\text { Price }\end{array}$ & Page Range & $\begin{array}{l}\text { Domestic } \\
\text { Price }\end{array}$ \\
\hline $001-025$ & $\$ 5.00$ & $326-350$ & $\$ 18.00$ \\
\hline $026-050$ & 6.00 & $351-375$ & 19.00 \\
\hline $051-075$ & 7.00 & $376-400$ & 20.00 \\
\hline $076-100$ & 8.00 & $401-425$ & 21.00 \\
\hline $101-125$ & 9.00 & $426-450$ & 22.00 \\
\hline $126-150$ & 10.00 & $451-475$ & 23.00 \\
\hline $151-175$ & 11.00 & $476-500$ & 24.00 \\
\hline $176-200$ & 12.00 & $501-525$ & 25.00 \\
\hline $201-225$ & 13.00 & $526-550$ & 26.00 \\
\hline $226-250$ & 14.00 & $551-525$ & 27.00 \\
\hline $251-275$ & 15.00 & $526-550$ & 28.00 \\
\hline $276-300$ & 16.00 & 601-up 1 & \\
\hline $301-325$ & 17.00 & & \\
\hline
\end{tabular}

${ }^{1}$ Add 2.00 for each additional 25 page increment from 601 pages up. 
Technical Information Department - Lawrence Livermore Laboratory University of California - Livermore, California 94550

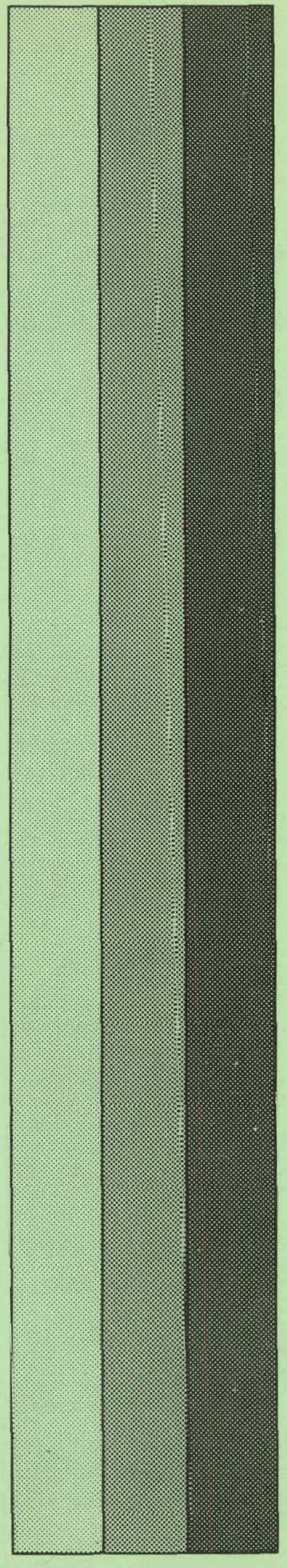

